\title{
Identification of Human CD4+ Sub-population of Resident Cardiac Fibroblasts Linked to Inflammation-Mediated Cardiac Fibrosis
}

Jamila H. Siamwala, $\mathrm{PhD}^{1,2}$; Francesco S. Pagano, BS ${ }^{1}$; Patrycja M Dubielecka, $\mathrm{PhD}^{3}$; Alexander Zhao, BS ${ }^{1}$; Sonja Chen, $\mathrm{MBBS}^{2,4}$; Haley Granston, BA ${ }^{1}$; Sharon Rounds, MD ${ }^{2,5}$ and Richard J. Gilbert, $\mathrm{MD}^{6}$

${ }^{1}$ Department of Molecular Pharmacology, Physiology and Biotechnology, Brown University, Providence, RI

${ }^{2}$ Warren Alpert Medical School of Brown University, Providence VA Medical Center, Providence, RI

${ }^{3}$ Division of Hematology/Oncology, Department of Medicine, Rhode Island Hospital, Warren Alpert Medical School of Brown University, Providence, RI

${ }^{4}$ Department of Pathology and Laboratory Medicine, Rhode Island Hospital, Providence, RI

${ }^{5}$ Division of Pulmonary, Critical Care, and Sleep Medicine, Department of Medicine, Warren Alpert Medical School of Brown University, Providence, RI

${ }^{6}$ Ocean State Research Institute, Providence VA Medical Center, Providence, RI

Running Title: $\mathrm{CD}^{+}$human cardiac fibroblast population

Key words: Inflammation, Cardiac Fibrosis, CD4 ${ }^{+}$, Transdifferentiation, Pulmonary Hypertension

\section{*Corresponding Author}

Jamila H. Siamwala, PhD

Department of Molecular Pharmacology, Physiology and Biotechnology, Brown University and VA Medical Center Providence, 830, Chalkstone Avenue, Building 35,

Providence, RI 02908

jamila_siamwala@brown.edu

The Warren Alpert Medical School of Brown University, Providence Veterans Affairs Medical Center,

830 Chalkstone Avenue,

Providence, RI 02908 


\section{ABSTRACT} available under aCC-BY-NC-ND 4.0 International license.

Background: Infiltration with inflammatory $\mathrm{CD} 4^{+} \mathrm{T}$-cells and the accumulation of heterogeneous cardiac myofibroblasts are hallmarks of cardiac fibrosis and remodeling. The origin, identity, states, and functions of the resident cells involved in the transition from adaptive to maladaptive fibrotic remodeling, as well as the pathways of inflammatory regulation are unclear.

Methods: We performed mass cytometry profiling of resident human ventricular cardiac fibroblasts (hVCF) and determined the identity of cells contained in fibrotic right ventricle autopsy tissues from individuals diagnosed with pulmonary hypertension and tissue from SUGEN/hypoxia rats exhibiting cardiac fibrosis. We further characterized the resident cardiac fibroblast sub-population morphologically, structurally and functionally using transcriptome and secretome analysis of the secreted cytokines, chemokines, proteins, metabolites using milliplex panels, proteomics and metabolomics pipelines.

Results: Single-cell mass cytometry identified remarkable plasticity of resident human cardiac fibroblasts. We provide evidence of a sub-population of resident cardiac myofibroblasts expressing high levels of $\mathrm{CD}^{+}$, a helper T-cell surface marker in addition to mesenchymal markers, $\alpha$ SMA and Vimentin in all the human donors. These cardiac cells co-expressing lymphoid $\mathrm{CD}^{+}$and $\alpha \mathrm{SMA}^{+}$were localized to the fibrotic regions of the human right ventricular tissue and were a common feature in the interstitial and perivascular lesions of SUGEN/Hypoxia (SuHx) rats. $\mathrm{CD}^{+} \mathrm{CD}^{+} \mathrm{T}$-cell numbers were higher in the right ventricle compared with the left ventricle of SuHx, as determined by flow cytometry. In vitro, T-cell homing receptors CD44, Interleukin-1 receptor (IL-1R), and CCR2 were upregulated in cardiac fibroblasts in response to IL-1 $\beta$. Exposure of cardiac fibroblasts to IL-1 $\beta$ led to upregulation of genes regulating extracellular matrix, collagen deposition and inflammation-related genes, and induced secretion of cytokines, chemokines, and metabolites involved in innate and adaptive humoral immune responses. Cell clustering, elevated phosphorylation of

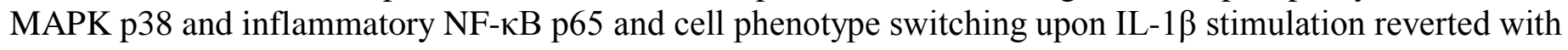
the administration of an IL-1R antagonist.

Conclusions: Our data expand concepts of heterogeneity of resident cardiac fibroblasts and plasticity in response to pro-inflammatory cytokines by the demonstration of a unique subpopulation of cardiac fibroblasts exhibiting attributes of both mesenchymal and lymphoid cells. Exposure of cardiac fibroblasts to the proinflammatory cytokine, IL-1 $\beta$, induces a robust phenotypic response linked to extracellular matrix deposition and up-regulates an immune-associated phenotype linked to expression of immune markers and secretion of immunomodulatory cytokines and chemokines. We also propose that resident cardiac fibroblast transdifferentiation and phenotype switching maybe the key process involved in adaptive to maladaptive remodeling leading to fibrosis and failure.

Non-standard abbreviations: $C D 4$; Cluster of differentiation, $\alpha S M A$; alpha smooth muscle actin, $I L$ $1 R$; Interleukin-1-receptor, $C C R 2$; C-X-C Motif Chemokine Receptor 2 


\section{INTRODUCTION}

Perivascular inflammation is frequently observed in lungs of patients with pulmonary hypertension $(\mathrm{PH})$ and plays a critical role in determining the prognosis of this disease ${ }^{1-5}$. Histological features of these lesions include perivascular inflammatory infiltrates involving $\mathrm{T}$ and B lymphocytes, macrophages, dendritic cells, and mast cells in the pulmonary arteries of both explanted human lungs and animal models of $\mathrm{PH}^{2,6}$. These lesions are linked to elevated plasma concentrations of cytokines derived from infiltrating immune cells, which serve as biomarkers of disease severity. However, the mechanism by which cytokines promote cardiac and vascular remodeling in human $\mathrm{PH}$ is unclear ${ }^{7}$. Among the cytokines reported to be elevated in $\mathrm{PH}$, interleukinn-1 (IL-1), is a multipotent, inflammatory agonist of cardiac fibroblast cells, which enhances chemotaxis and adhesion molecule expression in response to myocardial injury ${ }^{8}$. Interleukin-1 $($ IL-1 $\beta$ ), a member of the IL-1 family, exacerbates tissue damage during acute and chronic injury by activating multiple signaling pathways, including phosphorylation of kinases, $\mathrm{Ca}^{2+}$ flux, cytoskeletal reorganization ${ }^{9}$, and the release of inflammatory cytokines/chemokines, matrix metalloproteases ${ }^{10}$ and nitric oxide ${ }^{11}$. IL-1 $\beta$ binds to the Interleukin-1 receptor (IL-1R) to promote the release of secondary inflammatory cytokines such as IL-6, IL-23, Tumor Necrosis Factor-Alpha (TNF $\alpha$ ), and Granulocyte Colony-Stimulating Factor (G-CSF) ${ }^{12}$. IL-1 may also drive the differentiation of T-cells into Th17 cells and effector cells to produce pathogenic cytokines, such as IFN $\gamma$, IL-17, and Granulocyte-Macrophage Colony-Stimulating Factor (GM-CSF) ${ }^{13}$. Blocking the cytokine receptor IL-1R with an IL-1R antagonist reduces PH in a rat monocrotaline model ${ }^{14}$. Similarly, the monoclonal antibody to IL-1 $\beta$, canakinumab, decreases inflammation in right ventricular failure and $\mathrm{PH}$ in clinical Phase IB/II pilot studies suggesting the critical role of IL-1 $\beta$ in PH linked inflammation ${ }^{15}$. While it is known that IL-1 $\beta$ is expressed by activated disease-derived fibroblasts ${ }^{16}$, it is uncertain how IL- $1 \beta$ modulates cardiac fibroblast function or contributes to the inflammatory or fibrotic response in right ventricular failure in PH.

Fibroblasts exhibit heterogeneity as their identity, origin, properties, signaling mechanisms, and response to environmental stimuli are not well defined ${ }^{17}$. Activated pulmonary myofibroblasts, for example, 
are posited to be the drivers of perivascular inflammation and assume an epigenetically altered pro-

inflammatory phenotype, secreting cytokines, chemokines, and recruiting macrophages in calf, rat, and human models of pulmonary arterial hypertension ${ }^{18}$. Recent research suggests that resident cardiac myofibroblasts exhibit heterogeneity and versatility in mediating the response to local myocardial injury ${ }^{18,19}$. For example, activated resident fibroblasts can differentiate into hyper-proliferative myofibroblasts expressing smooth muscle cell actin ( $\alpha$ SMA) and secreting collagen, fibronectin, and other extracellular matrix molecules ${ }^{20,21}$. They can also be reprogrammed into cardiomyocyte-like cells using transcription factors and miRNAs. ${ }^{22,23}$. However, the responses of resident cardiac fibroblasts to myocardial injury and associated pro-inflammatory signals and their role in myocardial inflammation are poorly understood. Accumulating evidence suggests that $\mathrm{CD}^{+} \mathrm{T}$ cells participate in the repair of the myocardium after infarction, similar to the cardiac myofibroblast ${ }^{24}$, by modulating collagen deposition, degradation, and formation of fibrous tissues ${ }^{25}$. In contrast to their reparative role, Treg cells may also acquire a pro-inflammatory phenotype, thereby contributing to adverse cardiac remodeling in mice with chronic ischemic heart failure ${ }^{26}$. Specifically, $\mathrm{CD} 4^{+} \mathrm{T}$ cells are involved in the transition from compensated cardiac hypertrophy to maladaptive cardiac remodeling and heart failure during chronic pressure overload ${ }^{27}$. Blockade of T-cell co-stimulation in a transverse aortic constriction (TAC) mouse model of pressure overload reduces severity and delays heart failure, suggesting a crucial role of CD4 ${ }^{+}$ T-cells in the progression of this disease ${ }^{28}$.

Here we present evidence for the existence of a unique population of cardiac fibroblasts expressing both mesenchymal markers ( $\alpha \mathrm{SMA}$ and vimentin) and immune $\mathrm{CD} 4^{+}$markers and residing in right ventricular tissue from patients diagnosed with $\mathrm{PH}$. The $\mathrm{CD}^{+}$expressing sub-population of $\alpha \mathrm{SMA}^{+} \mathrm{Vimentin}^{+}$cardiac fibroblasts is confirmed in rats treated with SUGEN/Hypoxia, which has been reported to cause PH and RV modeling ${ }^{29-34}$. Our data also show that cultured human cardiac fibroblasts respond to IL-1 $\beta$ by transdifferentiating to lymphoid-lineage cells that modulate innate and adaptive immunity. These findings suggests that phenotype-altering responsiveness of cardiac fibroblasts to immunomodulatory agents may be important in the pathogenesis of cardiac fibrosis associated with pulmonary hypertension. 


\section{METHODS}

The authors declare that all supporting data are available within the article. Detailed methodology is provided in the online-only supplementary data. Antibodies used are listed in Supplementary Tables S2 and S3. The RNA Seq data are uploaded on the gene expression omnibus (GEO) database. The proteomics data are available in Proteomics IDEntifications (PRIDE) database at EMBL-EBI, a standardized public repository for protein and peptide identification.

Human right ventricle tissue and human cardiac fibroblasts. Institutional Review Board (IRB) approval for the use of human tissue was obtained from the Institutional Review Board of the Providence VA Medical Center. De-identified transverse right ventricular tissue sections of $10 \mu \mathrm{M}$ thickness were obtained post-mortem from patients with a diagnosis of $\mathrm{PH}$ and control tissue samples were obtained from patients who died of other causes from the Yale Tissue Repository Service, New Haven, Connecticut. Autopsy diagnoses of donors are described in Supplementary Table S4. Human ventricular cardiac fibroblasts (hVCF) were obtained from Lonza (Walkersville, MD) (Cat\# CC-2904) with the following lot numbers (Lot\# 67771, 62122, 1281202,534282 , TL210281) and a purity of $>99 \%$. Characteristics of donors of human cardiac fibroblasts are described in Supplementary Table S1.

\section{Animal models of PH}

\section{SUGEN/Hypoxia Rat Model of Cardiac Fibrosis and Primary Rat Ventricular Cardiac}

Fibroblasts. The animal experiments were approved by the Institutional Animal Care and Use Committee (IACUC) of the Providence VA Medical Center. Twenty male Fischer (CDF) rats (F344/DuCrl) weighing 220-250g were obtained from Charles River Laboratories. The animals were divided into two groups:

Normoxia $(\mathrm{Nx} ; \mathrm{n}=10)$ and SUGEN/Hypoxia $(\mathrm{SuHx} ; \mathrm{n}=10)$. Rats were administered a single intraperitoneal injection of the VEGFR inhibitor, SUGEN 5416 (Cayman Chemical; 20mg/kg). The animals in the SUGEN/Hypoxia group were then exposed to 3 weeks of normobaric hypoxia $\left(10 \% \mathrm{FiO}_{2}\right.$, Biospherix, Ltd, Parish, NY), followed by 5 weeks of normoxia ${ }^{29,35}$. The animals in the control group received a single 
intraperitoneal injection of the vehicle and were exposed to normoxia for the entire duration of the study ( 8

weeks in total). Fulton's index, a weight ratio of the RV divided by the sum of left ventricle and septum was measured to determine the extent of right ventricular hypertrophy. Cardiac fibrosis was determined by Sirius red staining and Masson trichrome staining in addition to Eosin and Hematoxylin staining. The images were analyzed using Orbit Image Analysis V3.15 software which can be trained to measure the entire section. Rat cardiac fibroblast cells were isolated from right and left ventricles (RV, LV) and processed for flow cytometry using a modified protocol published by Ackers-Johnson et $\mathrm{al}^{36}$. A detailed protocol is available in the onlineonly Data Supplement.

Statistical analysis. The normal or Gaussian distribution for small sample sizes was determined using Shapiro-Wilk's test. For statistical comparisons between 2 groups, 2-tailed unpaired $t$ test was used for unequal variances. When the sample sizes were small and not normally distributed, non-parametric tests 2-tailed MannWhitney U test was performed. Comparisons of $>2$ groups were made with 1- or 2- way ANOVA with either Tukey's post-hoc test as stated in the figure legends. Kruskal-Wallis tests followed by Dunn test comparison was used for continuous variables that did not show normal distribution. The values $* P<0.01$ and $* P<0.05$ was considered significant. The analyses were performed in GraphPad Prism version 8.0. Missing data are common in both proteomics and metabolomics due to heterogeneous responses to treatment or if the abundances of the protein or metabolite is below the detection limit of the mass spectrometer or a reduction in the electrospray performance. We used the Xia et al approach to disregard the variables containing missing data for our statistical analysis and focusing on the metabolites where the information was present for all the three human donors in both vehicle and IL-1 $\beta$ groups ${ }^{37}$. In some cases where there was a single missing value in one of the replicates, imputation using mean values were performed to replace the missing values. To control for multiplicity of the data, we employed the false discovery rate or Bonferroni correction as indicated. 


\section{RESULTS} available under aCC-BY-NC-ND 4.0 International license.

\section{Single-cell multidimensional mass cytometry identifies distinct cardiac fibroblast sub-populations}

expressing mesenchymal markers and lymphoid. To capture the human primary ventricular cardiac

fibroblasts (hVCF) heterogeneity and functional diversity, we performed multi-parametric

immunophenotyping using mass cytometry (Figure 1A) on primary human cardiac fibroblasts expressing

prototypical fibroblasts markers $\alpha$ SMA, vimentin, collagen-1, FSP-1, PDGFR $\beta$, and periostin and negative for

endothelial cell marker VE-cadherin (Figure S1) ${ }^{38-41}$. PDGFR $\beta$ positive cells were negative for FSP-1 and

some of the FSP-1 positive cells were negative for PDGFR $\beta$ (Figure S1). To determine the immune identity of the resident hVCF's, we categorized the cell sub-populations by barcoding the cells with heavy metal immune cell lineage specific antibodies and separating cell masses by time of flight (Figure 1A). Cisplantin, a cell viability stain was included in the panel of antibodies selected for sub-categorization of primary human cardiac fibroblast cells. The signals integrated per cell and the marker intensity and distribution were visualized using dimensionality reduction and clustering algorithms. First, the cell populations were cleaned by removing the beads and gated for live, nucleated single cells cell populations (Figure S2). Dimensionality reduction performed using viSNE analysis and T-distributed Stochastic Neighbor Embedding (tSNE) plots showed specific clusters of hVCF expressing $\alpha \mathrm{SMA}^{+}$and Vimentin ${ }^{+}$and similar levels of $\mathrm{CD}^{+}$, a hallmark helper-Tcell marker constituting $2.8 \% \pm 0.15 \%$ of the total analyzed cell populations (Figure 1B and Figure S3). Further, this population expressed other lymphoid cell markers such as CD4, CCR6, CD183 (Figure 1C) or CD45RA, CD45, CD16 (Figure S3) confirming the lymphoid lineage of the newly identified sub-population of cardiac fibroblast cells. Metaclusters generated using the FLOWSOM algorithm show three distinct populations of myeloid like cells, lymphoid like cells and dendritic cells (Figure 1G, Figure S4). In addition, higher resolution clustering using uniform manifold approximation and projection (UMAP) non-linear dimensionality reduction identified four primary human ventricular cardiac fibroblast subsets of lymphoid cells, myeloid cells, mesenchymal cells and dendritic cells (Figure 1D). To determine the frequency of activated cardiac fibroblast cells marked by $\alpha \mathrm{SMA}^{+}$expressing $\mathrm{CD}^{+}$and resident cardiac fibroblast cells 
marked by Vimentin expressing CD $4^{+} \mathrm{hVCF}$, we next gated cells expressing Vimentin ${ }^{+} \alpha \mathrm{SMA}^{+}$(Figure S5),

Vimentin $^{+} \alpha \mathrm{SMA}^{-}$(Figure S6) or Vimentin $\alpha \mathrm{SMA}^{+}$(Figure S7). Of the total population of hVCF cells

expressing $\mathrm{CD}^{+}, 29.6 \%$ of the total cells counted were Vimentin ${ }^{+} \alpha \mathrm{SMA}^{+} \mathrm{CD} 4^{+}, 11.91 \% \pm 2.32 \%$ cells of the total cells were Vimentin ${ }^{+} \alpha \mathrm{SMA}^{-} \mathrm{CD} 4^{+}$resident cardiac fibroblasts and $10.53 \% \pm 2 \%$ of the total cells were $\alpha \mathrm{SMA}^{+}$Vimentin ${ }^{-\mathrm{CD}^{+}}{ }^{+}$activated cardiac fibroblasts (Figures 1E, 1F). The total percentages of CD4+ subpopulations expressing either $\alpha$ SMA or Vimentin or both are summarized in Figure S8. However, cardiac fibroblast cells expressing triple markers Vimentin ${ }^{+} \alpha \mathrm{SMA}^{+} \mathrm{CD}^{+}$are about $3 \%$ of the total primary human ventricular cardiac fibroblast populations analyzed. Moreover, resident cardiac fibroblast expressing CD4 marked by Vimentin ${ }^{+}$cells were confirmed by immunostaining (Figure 1H). To exclude the possibility of contaminating T-cells in primary cultures, the cells were cultured in fibroblast growth media without T-cell activators for at least two to three passages. Besides size based exclusion, adherent cardiac fibroblasts were washed several times to ensure any loosely adhered immune cells were removed. Taken together, our data evidences the existence of a previously unidentified resident cardiac fibroblast sub-population that co-express dual mesenchymal Vimentin ${ }^{+} \alpha \mathrm{SMA}^{+}$and the lymphoid $\mathrm{CD} 4^{+}$marker.

\section{Distribution and expression of spindle shaped $\alpha \mathrm{SMA}^{+} \mathrm{CD}^{+}$co-expressing cells in the fibrotic}

right ventricle (RV) of patients with Pulmonary Hypertension (PH). Following the identification of CD4 ${ }^{+}$ human ventricular cardiac fibroblasts (hVCF) sub-population in vitro using mass cytometry, we determined the relevance of these cells to cardiac fibrosis and PH. The autopsy diagnoses of human RV tissue donors in the groups $\mathrm{PH}$ and no PH are provided in Table. S4. Morphological characterization of RV tissue using Hematoxylin and Eosin (H\&E), Sirius Red and CD4 or $\alpha$ SMA/CD4 immunostaining of RV tissue showed RV hypertrophy and fibrosis in both the $\mathrm{PH}$ and no PH groups. We also noted variable amounts of cardiac myocyte hypertrophy and age associated fibrosis in interstitial, perivascular, and sub- endocardial locations in the H\&E stained and Sirius red sections of all cases (A, B, C, D, E, and F) (Figure 2A). Interstitial fibrosis was observed within the posterior papillary muscle of the right ventricle. Endocardial surfaces of right ventricle, showed 
hypertrophic changes with big box car nuclei. Donor A demonstrated hypertrophic cardiac myocytes with box car nuclei and mild interstitial and perivascular fibrosis, highlighted by Sirius Red. Donor B demonstrated hypertrophic cardiac myocytes with marked sub-endocardial and interstitial fibrosis with entrapped cardiac myocytes in the areas of fibrosis. Cardiac myocytes surrounding the area of dense fibrosis were notably hypertrophied. Sirius Red staining highlighted extensive sub-endocardial fibrosis and interstitial fibrosis.

Donor $\mathrm{C}$ demonstrated hypertrophic cardiac myocytes with sub-endocardial and patchy interstitial fibrosis.

Sirius Red highlighted the bands of interstitial and sub endocardial fibrosis and appeared focally pericellular in areas. Donor D demonstrated fewer hypertrophic cardiac myocytes with scattered foci of interstitial fibrosis. Sirius Red staining of the interstitial fibrosis highlighted not only the larger areas of fibrosis but pericellular fibrosis, surrounding individual cardiac myocytes. Finally, Donor E demonstrated predominately perivascular fibrosis, which is highlighted by the Sirius Red stain (Figure 2A).

Cells with a spindle-shaped morphology and membranous $\mathrm{CD}^{+}$staining, were present in the perivascular connective tissue in cases with minimal interstitial fibrosis and in the dense fibrous tissue seen in some cases (Figure 2A). Cells with a round shape and membranous $\mathrm{CD}^{+}$staining typical of traditional T-cells were predominantly identified inside capillaries (Figure 2A, Donor F). Moreover, we found significant increases in spindle-shaped cardiac fibroblast expressing both $\alpha$ SMA and CD4 in the fibrotic regions compared to the non-fibrotic regions of RV tissue in humans with a clinical diagnosis of right heart hypertrophy and dilation compared to RV's from those without an autopsy diagnosis of PH (Figure 2A). The RV collagen content tended to increase in donors with a diagnosis of PH (Figure 2A). These results demonstrate the diversity within the population of cardiac fibroblast cells and suggest the existence of CD4 ${ }^{+}$ subpopulation of cardiac fibroblast in the fibrotic regions of the right ventricular myocardium.

\section{Distribution and expression of spindle shaped $\alpha \mathrm{SMA}^{+} \mathrm{CD}^{+}$co-expressing cells in the fibrotic}

right ventricle in rats treated with SUGEN/hypoxia. After determining the distribution of $\alpha \mathrm{SMA}^{+} \mathrm{CD}^{+}$in the fibrotic regions of human right ventricular tissue, we next sought to further examine the percentage of these 
cell populations in RV and LV using a well-established rat SUGEN/hypoxia (SuHx) model of PH . We utilized

Fischer rats, genetically prone to right heart failure and exhibit RV remodeling in severe $\mathrm{PH}$ as a model of cardiac fibrosis in our experiments ${ }^{29}$. Fischer rats were treated with a single intraperitoneal injection of VEGF inhibitor SUGEN (25mg/kg) and subjected to 3 weeks of hypoxia (Hx) followed by 5 weeks of normoxia (Nx) ${ }^{37}{ }^{36}$. Control animals were injected with vehicle and housed in room air or Nx for 8 weeks. The SUGEN/Hypoxia had lower body weights (Figure S10, Panel C). Although the Fulton index measured from the RV and LV weights using the formula $(\mathrm{RV} / \mathrm{LV}+\mathrm{S})$ was not significantly increased, there was echocardiographic evidence of PH in that TAPSE was significantly decreased in SUGEN/Hypoxia treated rats (Figure S10, Panel C). None of the other cardiovascular parameters measured using echocardiography, such as cardiac output, ejection fraction, and mean pulmonary arterial pressure, were significant. We noted significant increases in expression of Collagen I/III in both perivascular and interstitial regions in RV and LV determined using Sirius red staining and Masson trichrome staining (Figure S10, Panel A \& Panel B). Collagen I/III content, was significantly higher in RV, but not in LV, in SuHx as compared to Nx rats determined from machine learning based quantification of the green color for collagen and red color for Sirius red from the entire heart section (Figure 2C). Further, changes in cardiac fibroblast number and localization in response to SuHx treatment was determined using a fibroblast marker FSP-1 (Figure S10D). Perivascular remodeling and increased collagen deposition were noted in the Masson trichrome stained rat RV tissue section (Figure S10, Panel B). To determine fibrotic changes in the collagen content and distribution of collagen in the RV, we compared the expression of Collagen $1 \alpha$ protein in SuHx and Nx rats by immunostaining and Sirius Red (Figure 2C). We noted a significant increase in collagen content in the RV of SuHx rats compared to Nx rats. Further, the collagen content in the RV of SuHx rats was significantly higher than the LV (Figure 2C). Next, we quantified the numbers of cardiac fibroblasts co-expressing $\alpha \mathrm{SMA}^{+} \mathrm{CD} 4^{+}$cells that may accompany fibrotic changes in the RV of Nx and SuHx rats using confocal microscopy. We found three types of cell populations: T-cells ( $\left.\alpha \mathrm{SMA}^{-} \mathrm{CD}^{+}\right)$, cardiac fibroblasts $\left(\alpha \mathrm{SMA}^{+} \mathrm{CD} 4\right)$, and cardiac fibroblasts expressing $\mathrm{CD} 4$ $\left(\alpha \mathrm{SMA}^{+} \mathrm{CD} 4^{+}\right)$in the $\mathrm{RV}$ of the rat (Figure $\left.2 \mathrm{C}\right)$. We noted two morphologies of traditional CD4 positive cells - 
round $\alpha \mathrm{SMA}^{+} \mathrm{CD} 4^{+}$and spindle-shaped $\alpha \mathrm{SMA}^{+} \mathrm{CD} 4^{+}$in the $\mathrm{RV}$ regions which were significantly increased in SuHx compared to Nx rats (Figure $2 \mathrm{C}$ ). The number of $\alpha \mathrm{SMA}^{+} \mathrm{CD} 4^{+}$cells trended to be higher in the perivascular fibrotic regions of the RV of SuHx rats compared to control (Figure 2C).

\section{Comparison of $\mathrm{CD3}^{+} \mathrm{CD4}^{+}$cardiac myofibroblast expression in the right and left ventricles of}

SUGEN/hypoxia model rats. To determine the percentage of resident cardiac fibroblasts expressing CD4 in response to SUGEN/Hypoxia ( $\mathrm{SuHx}$ ), we quantified the percentages of $\mathrm{CD} 3^{+} \mathrm{CD} 4^{+}$adult rat primary cardiac fibroblasts populations isolated from the RV and LV of Nx and SuHx rats using flow cytometry (Figure 3A). The isolation method includes size based separation of cardiac fibroblasts from immune cell and elimination of red blood cells (RBC's). Single, viable cells were gated and 2D plots were constructed for both the RV and LV cardiac fibroblast populations. $\mathrm{CD}^{+} \mathrm{CD}^{-}$population was elevated in $\mathrm{RV}$ under both $\mathrm{Nx}(16.39 \% \pm 14.06 \%)$ and SuHx conditions $(17.24 \% \pm 15.46 \%)$ at comparable levels and was found to be less prominent in LV, under both $\mathrm{Nx}(2.10 \% \pm 0.71 \%)$ and SuHx conditions $(9.41 \% \pm 6.04 \%)$ (Figure 3B). In the LV, the percentages of $\mathrm{CD}^{+} \mathrm{CD}^{-}$cardiac fibroblasts were higher in the SuHx conditions and trending towards significance compared to Nx conditions $(2.10 \% \pm 0.71 \%$ vs $9.41 \% \pm 6.04 \%, \mathrm{p}=0.053)$. Percentages of $\mathrm{CD}^{+} \mathrm{CD}^{+}$populations expressing cardiac fibroblast cells showed some increase in $\mathrm{LV}(5.79 \% \pm 4.92 \%)$ under SuHx conditions in comparison to $\mathrm{Nx},(5.50 \% \pm 5.75 \%)$ although it did not reach significance (Figure 3B).In the $\mathrm{RV}$, there is no change in the percentage of $\mathrm{CD}^{+} \mathrm{CD}^{-}$cardiac fibroblast population after treatment with SuHx. In the $\mathrm{LV}$, there is an increase in the percentage of $\mathrm{CD}^{+} \mathrm{CD}^{-}$cardiac fibroblast population after treatment with SuHx (Figure 3B).

\section{IL-1 $\beta$ plays critical roles in proliferation and differentiation of primary human cardiac}

fibroblasts. Identification of $\mathrm{CD}^{+}$expressing cardiac fibroblasts in both the human and rat $\mathrm{RV}$ prompted us to explore possible mechanisms of induction of CD4 expression in cardiac fibroblasts. PH patients have high levels of plasma IL-1 $\beta$, that correlate with the severity of $\mathrm{PH},{ }^{15,42}$ and reduction of IL- $\beta$ reduces inflammation and improves right heart function ${ }^{15}$. Based on published literature ${ }^{43-46}$, we postulated that pro-inflammatory 
cytokine IL-1 $\beta$ may contribute to the induction of expression of CD4 in resident cardiac fibroblasts. The heat map provides an overview of cardiac fibroblasts expressing immune cell markers at varying intensities. (Figure S12, Panel A). In addition, heat maps suggest that CD4 expression increases with IL-1 $\beta$ treatment (Figure S12, Panel B). The non-redundancy scores suggests that CD4 is expressed at similar levels to vimentin and $\alpha$ SMA, markers of cardiac fibroblasts (Figure S12, Panel C). The percentage of HLA-DR ${ }^{+}$dendritic cell, CD4 ${ }^{+}$ lymphocyte, and $\mathrm{CD} 68^{+}$monocyte populations shift with IL-1 $\beta$ treatment (Figure S12, Panel D). $\alpha$ SMA, Vimentin, and CD4 expressions show a trend towards increasing with IL-1 $\beta$ treatment (Figure S12, Panel E).

To determine whether IL-1 $\beta$ mediates the fibrotic responses by stimulating cardiac fibroblast proliferation, $\mathrm{hVCF}$ were treated with vehicle or recombinant IL-1 $\beta$ for $24 \mathrm{~h}$ followed by BrdU and MTT assays and Ki67 staining. IL-1 $\beta$ treatment resulted in a statistically significant increase in cardiac fibroblast proliferation in a dose-dependent manner (Figure 4A). There was no difference in the cell proliferation rate of cardiac fibroblasts with IL-1 $\beta$ with time (Figure 4B). IL-1 $\beta$ similarly affected the proliferation rates of primary hVCF cells isolated from males and females (Figure 4C). IL- $1 \beta$ treatment trended towards stimulation of deposition of Collagen I $\alpha$ determined from total collagen in the lysates (Figure 4D) and confocal microscopy (Figure S13); however, statistical significance was not noted. To determine whether IL$1 \beta$ differentiates the cardiac fibroblasts and induces phenotype changes, human hVCF were treated with IL$1 \beta$ for $96 \mathrm{~h}$. IL-1 $\beta$ treatment induced significant changes in the morphology of hVCF cells by day 4 including increased detachment and rounding of cells (Figure 4E). Round cells with non-homogeneous DAPI-stained nuclei appeared with $96 \mathrm{~h} \mathrm{IL-1 \beta}$ treatment (Figure 4E). The round cells, but not the surrounding cells, were positive for both $\alpha \mathrm{SMA}$ and $\mathrm{CD} 4^{+} \mathrm{T}$ cells (Figure $4 \mathrm{E}$ ). The quantification of round cells shows a significant increase in these cells for all the male and female donors with IL-1 $\beta$ treatment for $96 \mathrm{~h}$ (Figure 4E). The rounded cells were viable and not apoptotic, as determined using trypan blue staining. We next characterized the subcellular phenotype switching of cardiac fibroblasts with IL-1 $\beta$ after $96 \mathrm{~h}$ using transmission electron microscopy. Transmission electron microscopy showed more prominent endoplasmic reticulum and golgi 
apparati, suggesting activation and hypersecretory transformation of cardiac fibroblasts in the presence of IL-

$1 \beta$ (Figure 4F). There were increased intracellular and budding extracellular microvesicles in the IL-1 $\beta$-treated cells. We sought to expand the number of cardiac fibroblasts with T-cell features by growing the cells in T-cell expansion media for 10 days with added IL-1 $\beta$ (Figure 4G). We noted the formation of cell clusters in all the human donors suggesting IL-1 $\beta$ induced changes in phenotype of cardiac fibroblasts day 7 onwards (Figure 4G).

\section{Transcriptome profiles of IL-1 $\beta \mathrm{CD4}^{+}$cell population demonstrate shifts from an adult} differentiated state to a stem-cell like de-differentiated state and re-differentiated lymphoidal cells. We flow sorted hVCF cells into CD4 ${ }^{+}$cardiac fibroblasts and $\mathrm{CD}^{-}$cardiac fibroblasts for both the Veh and IL$1 \beta$ groups after 10 days of differentiation in T-cell media and then characterized the gene expression signatures of the newly identified $\mathrm{CD}^{+}$expressing cardiac fibroblast using next generation deep total RNA sequencing (Figure S14). We noted both the unique signatures of IL-1 $\beta$ CD4 $4^{+} \mathrm{hVCF}$ population (5A and 5B) and differential gene expression between Veh and IL-1 $\beta$ groups (Figure 5C). Heatmaps based on unhierarchial clustering showed differences in transcriptomic signatures for the IL-1 $\beta \mathrm{CD}^{+}$and IL-1 $\beta \mathrm{CD}^{-}$population (Figure 5A). However, there were only 6 genes that were unique to Veh $\mathrm{CD}^{+}{ }^{+}$compared to Veh $\mathrm{CD}^{-}$group. Venn diagrams further showed that there were no genes shared by the newly identified Veh CD4 ${ }^{+}$and IL1 $\beta$ $\mathrm{CD}^{+}$populations. On the other hand, 255 genes were unique to IL-1 $\beta$ group (Figure $5 \mathrm{~B}$ ). The number of significant genes common to all the four groups were represented by the volcano plot (Figure 5C). Notably, there were 22 genes that were significantly down-regulated and 31 genes that are significantly upregulated in the IL-1 $\beta \mathrm{CD}^{+}$population. The $\mathrm{CD}^{+}$cardiac fibroblast population differed from the parent population in possessing a stem-cell like activated state, as evidenced by the presence of genes associated with pluripotency and reprogramming (WNT9B, SHC4, CER1, SOX2, SHISHA3, DLX2, SPATA9 and FOXA2). Moreover, CD4 ${ }^{+}$ stem cell-like cells are secretory unlike the $\mathrm{CD}^{-}{ }^{-}$cells as evidenced by the expression of micro vesicle and exosomes associated genes (CCR7, CRP, ACTA1, AZU1, CAECAM 8, CHMP4C, GSTA5, CRISP3, 
$H I S T 1 H 2 B C$ and REEP2). In addition, these stem cell like secretory cells are metabolically active (CCR7, CRP, ACTA1, AZU1, CEACAM8, CHMP4C, GSTA5, CRISP3, HIST1H2BC and REEP2), and possess the characteristics of lymphoidal cells (FOXP3, CD3D, IFNL4, TMIGD2, ILDR2, CXCL11, ICOS, TNFRSF18, FCAMR, PRKCQ, SIT1, CCL16 and NLRP10) as annotated based on their biological function using Database for Annotation, Visualization and Integrated Discovery (DAVID) (Figure 5D). Innate immunity linked genes (2'-5'-oligoadenylate synthase like (OASL), absent in melanoma 2 (AIM2)) and adaptive immunity linked genes (CD1c molecule, CD207 molecule), co-stimulatory signal during T-cell activation (inducible T-cell costimulatory $(I C O S))$, T-cell chemotaxis (CXCL11), TNF receptor superfamily member (TNFRSF18) involved in leukocyte migration, B-cell proliferation genes (GRB2 binding adaptor protein) and genes associated with phagocytosis (carcinoembryonic antigen related cell adhesion molecule 4 (CEACAM4) are unique to the IL-1 $\beta \mathrm{CD}^{+}$group. These experiments show that a small population of terminally differentiated resident cardiac fibroblasts can be de-differentiated to a stem cell like state and re-differentiated into lymphoid like cells in the presence of IL-1 $\beta$.

\section{IL-1 $\beta$ mediates the transcription of extracellular matrix genes and inflammatory genes. One of}

the key functional features of immune cells is the ability to secrete cytokines, chemokines and immunomodulatory proteins. To determine whether the cardiac fibroblasts associated extracellular matrix and inflammation associated immune genes are activated by IL-1 $\beta$, we used a qPCR approach of gene expression quantitation using validated taqman array plate preset with primers specific to extracellular matrix genes or inflammation genes. A comprehensive list of both extracellular matrix genes and inflammation genes modulated by IL-1 $\beta$ is presented as a heatmap (Figure S15, Panel A and Panel B) and as a supplementary table (Table S6 and Table S7). The IL-1 $\beta$ responsive and unresponsive inflammatory and extracellular matrix genes are listed from highest Z-score to the lowest Z-score. (Figure S15, Panel A and Panel B). Notably, CD44 gene, highly expressed during T-cell development is upregulated by 3 folds in IL-1 $\beta$ treated cardiac fibroblast cells (Table S7). Further relationships between genes including activation, inhibition, binding partners of genes that 

available under aCC-BY-NC-ND 4.0 International license.

are differentially expressed with IL-1 $\beta$ were represented by string analysis (Figure S15, Panel C). The

inflammation gene expression data analyzed using string predicts IL-1R1 interaction with $N F k B 1$. In addition, $A N X A 1$ which is modulated with IL-1 $\beta$ is predicted to interact with multiple binding partners such as PTAFR, LTB4R and HRH1. The extracellular matrix gene expression data shows a cluster of ITGA modulated by IL$1 \beta$ interacting with binding partners and CD44 interacting with $M M P 14$ and VCAN. MMP1 is predicted to interact with MMP11 and $A 2 M$ (Figure S15, Panel C). Further, pathway analysis using CYTOSCAPE suggested that immune responsive genes with false discovery rate (FDR) of $2.85 \times 10^{-13}$ belonged to gene ontology terms "cellular response to cytokine stimulus", "cytokine-mediated signaling pathway"," inflammatory response", "defense response and immune system process" (Figure S15C). While the extracellular matrix associated genes with the FDR of $2.13 \times 10^{-17}$ belonged to gene ontology term “extracellular matrix organization”. We further validated the gene expression of (CCR2, CCL2, TGFB, IL-1R) (Figure S15E). MMP-9 trended toward an increase in hVCF and Col II-III trended toward a decrease after treatment with IL-1 $\beta$ (Figure S15E).

Induction of immunomodulatory proteins. One of the key functional features of immune cells is the ability to secrete cytokines, chemokines and immunomodulatory proteins. To determine the effect of IL-1 $1 \beta$ on the cardiac fibroblasts secretome (immunomodulatory cytokines, chemokines, proteins and metabolites), we performed quantitative cytokines and, chemokine analysis, proteomics, and metabolomics. IL-

$1 \beta$ dependent cytokine and chemokine secretion in the media was analyzed using MILLIPLEX MAP Human Cytokine/Chemokine Magnetic Beads in the cardiac fibroblasts from three human donors. We noted that $24 \mathrm{~h}$ treatment with IL-1 $\beta$ significantly increased secretion of IL-8 and IL-10, and trended to increase secretion of IL-6, MCP-3, IL-12p70, TNF- $\alpha$, and VEGF and trended to decrease IL-1 $\beta$ levels, although the results were not statistically significant (Figures S16, Panel B). Unlabeled proteomics using LC MS/MS to identify secreted proteins showed total of 525 and 588 proteins secreted in the conditioned media of cardiac fibroblast treated with Veh or IL-1 $\beta(10 \mathrm{ng} / \mathrm{mL})$ (Figure S17A, Tables S8-S11). The Venn diagram demonstrated that out of the 
annotated 394 proteins, 120 proteins were unique to IL-1 $\beta$ and 181 proteins common to both Veh and IL-

$1 \beta$ (Figure S17B). The conditioned media replicates for Veh and IL-1 $\beta$ groups were fairly consistent internally as represented in Figure S17C. In Figure S16, Panel D, the gene ontology terms that were synonymous with functions of secreted proteins with-Log (Benjamini-Hochberg) less than $\mathrm{p}<0.001$ are listed showing the proteins involved in extracellular matrix regulation and inflammation are secreted by hVCF in response to IL$1 \beta$. Tables S6 to Table S9 summarizes the functions of proteins in terms of biological processes, molecular function and immune subset population identified uniquely in the conditioned media of IL-1 $\beta$ treated hVCF. Gene Ontology (GO) based annotation of proteins uniquely found in the conditioned media from cardiac fibroblasts treated with IL-1 $\beta$ (Tables S6-S9) showed that these secreted proteins were involved in the activation of both adaptive and innate immune responses (GO: 0042102 Positive regulation of T-cell proliferation, GO: 0006959 Humoral immune response, GO: 0002443 Leukocyte mediated immunity, GO: 0002252 Immune effector process, GO: 0002576 Platelet degranulation, GO: 0006956 Complement activation, GO: 0002274 Myeloid leukocyte activation and GO: 0002446 Neutrophil mediated immunity) and recruitment of inflammatory and reparative immune cells (CXCL1, CCL2, CXCL3, CXCL5,CXCL8 apolipoprotein A1, IL-6, complement factor H related (CFHR1), VCAM1). Specifically, proteins involved in the activation of innate and adaptive immune responses, leukocyte activation, and leukocyte migration were displayed in the biological process analysis using PANTHER (Figure S17).

Cytokine induced secretion of immune system associated metabolites. We next sought to analyze the metabolites in the conditioned media following treatment with IL-1 $\beta$ to determine the overall changes in the metabolic status of cardiac fibroblasts using non-targeted metabolite profiling of the conditioned cell culture media. Principal component analysis based on overall differences between the metabolites indicate some separation of vehicle group and IL-1 $\beta$ treated groups (Figure S18, Panel A). The volcano plot distinguished metabolites with negative log with a false discovery rate higher than 1 modulated by IL$1 \beta$ treatment (Figure S18, Panel B). Heatmap comparisons of differentially secreted metabolites between 
vehicle and IL-1 $\beta$ are presented in Figure S18, Panel C. Interestingly, the metabolic profile for the female donor of the cardiac fibroblast cells with and IL-1 $\beta$ treatment was different from the male donors (Figure S18, Panel C). The top 25 differentially upregulated and downregulated metabolites are represented on the heat map (Figure S18, Panel D). Pathway analysis of the differentially expressed metabolites indicated that metabolites involved in cardiolipin biosynthesis, a key inner mitochondrial membrane protein and mitochondrial oxidant producer, were significantly upregulated in IL-1 $\beta$ exposed cardiac fibroblast cells (Figure S18, Panel E). Typical CD4 ${ }^{+}$metabolite signatures of activated $\mathrm{T}$ cells involving methionine metabolic pathways and pyrimidine metabolism were also upregulated. Methyl histidine metabolism, phenylacetate metabolism, and de novo triacyglycerol biosynthesis were also upregulated (Figure S18E). Interestingly, metabolites serving as a link between glycolysis and citric acid cycle including thiamine pyrophosphate were differentially secreted in response to IL-1 $\beta$ stimulation. Specifically, metabolites associated with ATP generation, carbohydrate metabolism, and the production of amino acids, nucleic acids, and fatty acids such as spermidine, spermine and atrolactic acid were upregulated, while others were down-regulated such as sedoheptulose-1-7-phosphate, oxaloacetate, N6-acetyl-L-lysine, succinate, 1,3 diphosphate glycerate, citrate-isocitrate (Figure S18F and Table S12-13). In sum, our data indicates that in the presence of IL-1 $\beta$ cardiac fibroblasts assume immune cell features by upregulating inflammatory and extracellular matrix genes, and secreting cytokines, chemokines, and immunomodulatory proteins and metabolites involved in both innate and adaptive immune response critical in both pathogenesis and resolution of injury.

\section{Transdifferentiation of cardiac fibroblasts into lymphoid cells is mediated by IL-1R-pMAPK and}

IL-1R-NFkB pathways. To determine the mechanisms of IL-1 $\beta$ mediated differentiation of cardiac fibroblasts into a cell with immune cell features, we measured the expression of Interleukin-1 receptor (IL-1R) on the cardiac fibroblasts. IL-1R gene expression increases significantly with IL-1 $\beta(10 \mathrm{ng} / \mathrm{mL})$ after $24 \mathrm{~h}$ treatment (Figure 6A). We also visualized a dramatic increase in IL-1R puncta both in the nucleus and cytoplasm as shown by confocal microscopy. In addition we noted secretion of the IL-1R outside the cells visualized using 
$3 \mathrm{D}$ rendering of the confocal images obtained from immunostained IL-1 $\beta$ treated hVCF (Figure $6 \mathrm{~B}$ ). In

addition, IL-1R protein expression and foci formation was significantly reduced with IL-1Ra $(1 \mu \mathrm{g} / \mathrm{mL})$.

Differentiation of cardiac fibroblasts in T-cell media for 14 days showed that the cell clusters typical of IL-1 $\beta$

treatment were significantly reduced with IL-1RA (Figure 6C). We next assessed the activation status of IL$1 \beta$ driven downstream signaling to pathways involved in proliferation and differentiation i.e. p38 MAPK and NF- $\kappa$ B p65 signaling pathway. We show that treatment with IL-1 $\beta$ increases pro-differentiation p38 MAPK phosphorylation at Thr180/Tyr182 residue (Figure 6D). This effect was blunted by incubation with IL-1RA, but not rescued by IL-1 $\beta$. Similar results were noted with NF- $\kappa$ B p65 phosphorylation at Ser 536 residue in 2 out of 3 instances and not reach statistical significance (Figure 6D).

\section{DISCUSSION}

Myocardial injury is characterized by complex interactions of mesenchymal and immune cells that collectively form the backbone of regenerative repair in the heart ${ }^{47,48}$. We identify a sub-population of hVCF expressing surface markers of both quiescent cardiac fibroblasts (Vimentin), activated cardiac fibroblasts ( $\alpha$ SMA) and lymphoid T-cells (CD4) using single cell mass cytometry. Further, we confirm the presence of these spindle shaped cells dually expressing $\alpha \mathrm{SMA}$ and CD4 in fibrotic areas of the right ventricles of humans with PH and right ventricles of rats subjected to SUGEN/hypoxia to model PH. Moreover, we demonstrate that this sub-population of resident cardiac fibroblast cells behave structurally and functionally similar to lymphoid immune cells when exposed to pro-inflammatory cytokine IL-1 $\beta$, and that they produce a prototypical fibroblast-immune secretome (genome, proteome, metabolome) related to extracellular matrix deposition and inflammation consistent with its proposed role in the construction of the myocardial inflammasome ${ }^{43,49}$ (Figure 7). Blocking of IL-1 receptor using an IL-1R antagonist reverses IL-1 $\beta$ mediated transdifferentiation of cardiac fibroblast cells and p-p38 MAPK phosphorylation. Lastly, characterization of the IL1- $\beta$ induced CD4 ${ }^{+}$ population using deep RNA sequencing suggests a shift from the differentiated state to a de-differentiated stem cell state and re-differentiated lymphoidal cell population. 
Fibroblasts display heterogeneity of form, function, and fate in response to varied activation signals, epigenetic alterations, phenotypic plasticity, and transdifferentiation ${ }^{50,51,40 .}$. Differentiated cardiac myofibroblasts exhibit hyperplasia and excess deposition of extracellular matrix that remodels the interstitium following injury, thereby affecting ventricular contractility and promoting systolic and diastolic dysfunction. Similar to macrophages, cardiac fibroblasts assume an inflammatory role by secreting immune cell- recruiting cytokines and chemokines ${ }^{52}$ and assume a phagocytic role ${ }^{53}$ suggesting phenotype plasticity and cell switching in the setting of myocardial injury.

A growing body of evidence suggests that $\mathrm{PH}$ is a syndrome with immune dysregulation involving the $\mathrm{CD}^{+}$helper T-cell type I (Th1)/Th17 immunity. Modulating CD4 ${ }^{+}$Treg cells reduces endothelial injury and prevents pulmonary hypertension in T-cell deficient rats ${ }^{54,55}$. Mature $\mathrm{CD} 4^{+}$depleted mice are protected from left ventricular fibrosis, but not cardiac hypertrophy in Transverse Aortic Constriction (TAC) induced left ventricular failure ${ }^{27}$. The mechanisms by which $\mathrm{CD}^{+}$Treg cells potentiate fibrosis is unclear. It is speculated that $\mathrm{CD}^{+}$Treg cells recruited from the bone marrow secrete extracellular matrix similar to cardiac fibroblasts which promotes thickening and reduces compliance of the ventricular wall, leading to systolic and diastolic dysfunction. Similarly, resident myofibroblasts may be activated or recruited in response to damage-associated molecular patterns (DAMPS) from the damaged myocytes to accelerate the process of extracellular deposition and healing; however, this theory is debated ${ }^{26}$. Yano et al suggest that resident cardiac fibroblasts, but not bone marrow derived cells, add to the myofibroblast pool in the setting of myocardial repair ${ }^{56}$. The surge in the numbers of myofibroblasts following myocardial injury is unaccounted for and the role of resident cardiac fibroblasts in myofibroblast accumulation is not known ${ }^{57}$. Recent attempts have been made to identify and trace the lineage of the sudden expansion and activation of fibroblast cell populations after myocardial infarction using periostin and TCF-21 mice models ${ }^{58,59}$. Results from these models demonstrate that circulating bone marrow derived cells, $\mathrm{CD} 14^{+}$fibrocytes, smooth muscle cells, and endothelial cells transform into cardiac myofibroblasts through the endothelial to mesenchymal transition process which maybe a source of myofibroblast accumulation and deposition of excess of extracellular matrix ${ }^{20,60-62}$. T-cell macrophage cell 

available under aCC-BY-NC-ND 4.0 International license.

fusion is common resulting in giant cells. We show the appearance of notch shaped non-homogeneous giant cells after treatment of cardiac fibroblasts with IL-1 $\beta$ for 4 days confirming yet another feature of T-cells

(Figure 4). Cell phenotype switching is complex and it is reasonable to expect more than a singular cell type or pathway is involved in this complicated inflammatory response. Our RNA seq data further suggests that terminally differentiated cardiac fibroblasts may de-differentiate into a stem-cell like metabolically active state and then re-differentiate into lymphoidal cells in response to IL-1 $\beta$, further opening up a new possibility of regenerating damaged cells through de-differentiation and transdifferentiation ${ }^{63}$. In fact, the epigenetic and metabolic reprogramming seen in the $\mathrm{CD}^{+}$population is reminiscent of induction of lymphocyte dependent adaptive immune responses and trained immunity ${ }^{64}$.

Compensatory transdifferentiation mechanisms of resident cells into immune-like phenotypes in a critical organ such as the heart may involve the orchestration of multiple cell types, growth factors, tissue repair agents, metabolite consumption and secretome release. As a result, the generation of a global knockout or cardiac fibroblast specific conditional knockout of IL-1 $\beta$ in mice may not be an ideal model to study the mechanism of in vivo transdifferentiation. Such a preparation would require a simultaneous knockout of multiple cytokine and chemokine factors. On the other hand, lineage tracing to follow the origin, fate and function of cardiac fibroblasts expressing dual mesenchymal and lymphoidal features has value and will be performed in our next phase of work.

There are some limitations to the work presented in this manuscript. We were limited in resources to demonstrate IL-1 $\beta$ mediated fibroblast trans-differentiation using lineage tracing in in vivo models of PH and RV failure. We note that mass cytometry was performed on non-permeabilized primary human ventricular cardiac fibroblasts. There are two versions of CD3, a cytosolic and cell surface antibody which is commonly used in the clinical pathology to stain human autopsy tissues. It is likely that the validated CD3 antibodies that we used would have worked with permeabilized cells to visualize the cytosolic CD3 expression and therefore absence of CD3 maybe a technical problem as opposed to a complete lack of expression in the 
transdifferentiated human cardiac fibroblast cells which was later confirmed from the CD3 expression in the RNA Seq data. Studies specifically considering the functional role of trans-differentiated myofibroblasts, lineage, fate, epigenetic changes, regulation, and interaction with surrounding cells are fundamental and will be the focus of future studies. We cannot exclude the involvement of other cytokines and chemokines in the transdifferentiation of the cardiac fibroblasts into lymphoid lineage in vivo. Our work, however, indicates that IL- $1 \beta$ alone is sufficient to transdifferentiate $2-3 \%$ of the cardiac fibroblast population into $\mathrm{CD}^{+}$cells possibly through de-differentiation into a stem cell like cell and re-differentiation. IL-1R downstream signaling mediators, promoter binding elements, and gene expression regulating molecules will be studied in the future. Lastly, we have not addressed the possibility that the process of transdifferentiation may be bidirectional, with phenotype switching from cardiac fibroblasts to lymphoid cells coupled with phenotype switching from lymphoid cells back to cardiac fibroblasts.

Our study proposes a new paradigm of inflammo-fibrosis whereby resident cardiac fibroblasts dedifferentiate into a multi-functional stem cell like cell and re-differentiate into $\mathrm{CD}^{+}{ }^{+}$cells in the presence of IL$1 \beta$, providing an opportunity to utilize plasticity conferred by IL-1 $\beta$ to replace cell loss and regenerate the heart $^{65}$. We propose a model wherein resident fibroblasts undergo staged cyclical polarization/phenotype switching in response to pro-inflammatory and anti-inflammatory signals in the tissue environment. We speculate that tissue-based cardiac fibroblasts, which transdifferentiate into CD4 immune cells, may function as "first responders" in advance of the recruitment of macrophages, natural killer (NK) cells, and neutrophils. Recognizing the potential role of fibroblast plasticity in orchestrating the conversion, enlistment, and activation of an array of inflammatory cell types to the stressed myocardium, therapeutic methods designed to modulate cardiac fibroblast plasticity related inflammation-induced right ventricular fibrosis may emerge in the future. Since right ventricular failure is the most common cause of mortality in $\mathrm{PH}$, modulation of fibroblast plasticity raises the possibility of new approaches to therapy by preventing transition from adaptive to maladaptive and failure and healing through re-differentiation of adult cells. 


\section{ACKNOWLEDGEMENTS}

The study was funded in part by grant P20 GM103652 from the National Institute of General Medical Sciences, National Institutes of Health to Sharon Rounds (SR) and Elizabeth O. Harrington (EOH), R01HL130230 (SR), Rhode Island Foundation grant (20190594) Alzheimer Supplement and TEAM UTRA grant from Brown University to JHS and NIH R56/R01 HL139680 to RJG. We thank Dr. Ruth Montgomery, PhD and Ms. Shelly Ren from the Yale for the CyTOF core facility and Dr. John M Asara, PhD, Director of the Mass Spectrometry core, Harvard Medical School for helpful discussions. We thank Siraj Presswala for his assistance with the UMAP analysis using PYTHON and R tools and Daniel Federick from Fluidigm for help with the tSNE analysis using CYTOBANK. We also acknowledge Nagib Ahsan and Lelia Noble from COBRE Center for Cancer Research Development, Proteomics Core Facility, Rhode Island Hospital, Providence, RI for their help with proteomic sample processing and analysis. The authors also acknowledge support from Dr. Fenghai Duan of advance CTR (U54GM115677) for help with the statistical analysis of proteomics and metabolomics data. We thank Yang Zhou for his help with the animal measurements. We would also like to acknowledge the Choudhary Lab at the Providence VA Medical Center for the rat blood and spleen to validate the CD4 antibodies using flow cytometry and immunostaining. We thank Gaurav Choudhary, MD and Elizabeth O. Harrington, PhD for helpful discussions. This work was performed using the facilities and resources of CPVB COBRE and Providence Veterans Affairs Medical Center.

\section{DISCLOSURES}

The authors declare no conflicts of interest. 


\section{FIGURE LEGENDS} available under aCC-BY-NC-ND 4.0 International license.

\section{Figure 1: Identification of a sub-population of resident hVCF expressing mesenchymal and lymphoid}

cell markers with multidimensional single cell mass cytometry. Immunophenotyping of hVCF derived from human subjects 2 males (ID\# 62122, ID\# 1281202) and 1 female (ID\#534282) was performed by staining 3 x $10^{6}$ cells with heavy metal tagged antibodies (Table S3) followed by mass cytometry to identify single-cell expression. T-distributed Stochastic Neighbor Embedding (tSNE) plots derived using viSNE algorithm showing population distribution and intensity of protein. (A) Schematic summary of mass cytometry analysis. hVCF were immune stained with epitope-specific antibodies conjugated to transition element isotope reporters of different masses. The cells are nebulized into single-cell droplets followed by acquisition of an elemental mass spectrum. Traditional flow cytometry methods are used to analyze integrated elemental reporter signals for each cell. (B) viSNE graphs of manually gated live, nucleated, hVCF cell clusters expressing varying marker intensities and distribution. The resident $\mathrm{hVCF}$ are marked by Vimentin expression and activated cardiac fibroblasts are marked by $\alpha$ SMA expression. (C) The lymphoid lineage proteins expressing cells are marked by the cluster of differentiation (CD) CD3, CD4, CCR6, and CD183 on hVCF. Protein expression levels are demonstrated on the secondary y-axis scale with blue showing no expression, green, the least expression and red showing the highest expression. Each dot represents the expression profile of a single cell.

(D) Preservation of the global structure using higher resolution Uniform Manifold Approximation and Projection (UMAP) analysis of the expression of fibroblast and immune cell marker further confirm the expression of lymphoidal markers on cardiac fibroblast cells and identify clusters of cells expressing myeloid markers and dendritic markers. (E) Mass cytometry 2D plots of percentages of hVCF population expressing quiescent cardiac fibroblast marker Vimentin and activated cardiac fibroblast cell marker $\alpha$ SMA. The red arrow from quadrant 1 indicate the percentage of $\mathrm{CD}^{+}$cells among the Vimentin ${ }^{-} \alpha \mathrm{SMA}^{+}$activated resident cardiac fibroblast population. The arrows from quadrant 3 indicate the percentage of $\mathrm{CD}^{+}$cells among the Vimentin $^{+} \alpha \mathrm{SMA}^{-}$quiescent resident cardiac fibroblast population. The arrows from quadrant 2 indicate the percentages of $\mathrm{CD}^{+}$cells among the Vimentin ${ }^{+} \alpha \mathrm{SMA}^{+}$population. (F) Quantification of $\mathrm{CD}^{+} \mathrm{CD}^{-}$cells 
gated from Vimentin ${ }^{+} \alpha \mathrm{SMA}^{-}, \mathrm{Vimentin}^{-} \alpha \mathrm{SMA}^{+}$and Vimentin ${ }^{+} \alpha \mathrm{SMA}^{+}$populations is represented as a scatter

plot of Mean $\pm \mathrm{SD}$ ( $\mathrm{n}=3$ biological replicates, 2 males. 1 female; $* P<0.05$ determined using one-way ANOVA and Tukey post-hoc multiple comparison. (G) Metaclustering of the populations based on the distances of neighbors (UMAP algorithm). The colors stratify the similar and dissimilar cell populations showing the heterogeneity. (H) Representative immunostained images of resident hVCF with CD4, Vimentin and DAPI antibodies showing co-expression of Vimentin and CD4.

\section{Figure 2: Distribution and expression of CD4 in the RV in cardiac fibrosis in humans and in the rat}

model of SUGEN/Hypoxia PH. (A) Human right ventricular tissue from 6 autopsied donor diagnosed with other conditions (Donor A, C and E) or PH (Donor B, D and F) were stained with standard hematoxylin \& eosin (H\&E) and connective tissue Sirius Red. Donors A-E were immunostained for CD4 and counterstained with methyl green to identify nuclei. Donor F was used as a negative control for CD4 immunostaining. The black arrows point to fibrotic areas in H\&E, Sirius Red and CD4 stained sections. CD4 ${ }^{+}$expressing spindle shaped cells in the RV of donor tissue were determined, with arrows pointing to $\mathrm{CD}^{+}{ }^{+}$spindle-shaped cells. Scale bars, $75 \mu \mathrm{M}$. Quantification of dual stained $\alpha$ SMA (red) and CD4 (blue) in the fibrotic and non-fibrotic regions of human RV tissue sections performed using immunohistochemistry procedure. Scale bars are $1 \mathrm{mM}$. $* P<0.05$, one-way ANOVA and Tukey multiple comparison test. Quantification of collagen I/III content in the human RV determined using Sirius red is represented as scatter plots of Mean \pm SD (n=3 donors, $P=0.0897$, one tailed unpaired $t$ test and Mann Whitney Analysis of Ranks, PH vs No PH). (B) Schematic representation of the SUGEN/hypoxia for simulating PH in rats. Male Fischer rats were given a single bolus of SUGEN $(20 \mathrm{mg} / \mathrm{kg})$ and exposed to hypoxia $\left(10 \% \mathrm{FiO}_{2}\right)$ for 3 weeks, followed by exposure to normoxia $(\mathrm{Nx})$ for an additional 5 weeks (SuHx). In parallel, control animals were exposed to Nx (room air) for 8 weeks. Each Nx and $\mathrm{SuHx}$ group had $\mathrm{n}=10$ rats. (C) Collagen $1, \mathrm{CD}^{+}$(green), $\alpha \mathrm{SMA}^{+}$(red) and DAPI (blue) immunostaining of optimal cutting temperature (OCT) sectioned RV ( $7 \mu \mathrm{m}$ thick) from the rats subjected to Nx or SuHx. Collagen content determined using Sirius red staining of the RV and LV sections from Nx and SuHx rats is 

available under aCC-BY-NC-ND 4.0 International license.

represented as scatterplots of Mean $\pm \mathrm{SD}(\mathrm{n}=10$ rats, $* P<0.05$, one-way ANOVA and Tukey multiple

comparison test). The representative images are presented in the Supplementary Figure 10. Quantification of cells co-expressing $\mathrm{CD}^{+} \alpha \mathrm{SMA}^{+}$are presented as average scatter plots of Mean $\pm \mathrm{SD}(\mathrm{n}=3)$. One tailed unpaired $t$ test and Mann Whitney Analysis of Ranks, PH vs No PH.

Figure 3: Analysis of $\mathrm{CD3}^{+} \mathrm{CD4}^{+}$cardiac fibroblast cell populations in the $\mathrm{RV}$ and $\mathrm{LV}$ of $\mathrm{SuHx}$ model of PH using flow cytometry. Quantification of $\mathrm{CD}^{+}$and/or $\mathrm{CD} 4^{+}$cardiac fibroblast populations in the $\mathrm{RV}$ and LV. (A) Gating strategy of cardiac fibroblasts isolated using non-Langendorff method of cell isolation from both the RV and LV of Nx and SuHx rat hearts ( $\mathrm{n}=4$ males). (B) Data is presented in 2D biaxial flow cytometry plots indicating $\mathrm{CD}^{-} \mathrm{CD}^{-}, \mathrm{CD}^{+} \mathrm{CD}^{-}, \mathrm{CD}^{-} \mathrm{CD}^{+}$, and $\mathrm{CD} 3^{+} \mathrm{CD}^{+}$populations (upper panels) in the RV and LV of Nx and SuHx rat hearts and as scatter plots (lower panel) of Mean \pm SD. n=4 rats, $P=0.053$ RV Nx vs LV Nx and $* P<0.05 \mathrm{Nx}$ vs SuHx. The red lasso suggests the emergence of a population from the parent population.

Figure 4: IL-1 $\beta$ mediated proliferation, transdifferentiation and activation of primary human ventricular cardiac fibroblasts (hVCF) into CD4 expressing cells in vitro. Primary hVCF cells $\left(2.0 \times 10^{6}\right.$ cells/mL) from donors (2 males (ID\# 62122, ID\# 1281202) and 1 female (ID\#534282) were cultured in Fibroblast Basal Media (FBM) for 4 days with 10ng/ml of IL-1 $\beta$ or in T-cell media with CD3/CD28 T-cell activator for 13 days. (A) hVCF proliferation in response to IL-1 $\beta$ exposure was assessed by BrdU incorporation. Data are Mean \pm SD for 3 biological replicates. $* P<0.05$, one-way ANOVA with multiple comparisons. (B) Response of hVCF to IL-1 $\beta$ after $24 \mathrm{~h}, 48 \mathrm{~h}$ and $72 \mathrm{~h}$ of incubation was assessed using MTT assay. Data are Mean \pm SD for 3 biological replicates ( $n=3-6$ technical replicates). (C) Cell proliferation of the 3 human subjects hVCF (ID\# 62122; male, ID\# 1281202; male and ID\#534282; female) in response to IL-1 $\beta$ was determined by Ki67 staining of nuclei. Data are Mean \pm SD for 3-4 biological replicates, $P=0.1$, one-way Kruskal-Wallis ANOVA with Dunn's multiple comparisons (D) Measurement of collagen content in response 
to IL-1 $\beta$ doses $(1 \mathrm{ng} / \mathrm{mL}$ and $10 \mathrm{ng} / \mathrm{mL})$ was measured in the cell lysates using Sircol assay. The data are represented as Mean \pm SD values from individual subjects. $(n=4)$ biological replicates, no significance (ns), Kruskal-Wallis one-way ANOVA with Dunn's multiple comparisons. (E) Left Panel: Bright field images of live hVCF cells indicating the shifts in cellular morphology from a spindle-shaped fibroblast to a round cell with a large nucleus in response to $96 \mathrm{~h}$ of treatment with IL-1 $\beta(10 \mathrm{ng} / \mathrm{mL})$. Arrows indicate a change in morphology of hVCF with IL-1 $\beta$ treatment, but not with vehicle treatment. Right Panel. Immunostaining of the fixed hVCF with lymphoid CD4 T-cell marker (green), mesenchymal aSMA (red) and DAPI (blue) markers to characterize the transformed cells. The arrow indicates the emergence of round, trans-differentiated, multinucleated $\mathrm{aSMA}^{+} \mathrm{CD} 4^{+}$expressing cells. Representative magnified images of multinucleated notch shaped giant immune cell immunostained with $\mathrm{aSMA}^{+} \mathrm{CD}^{+}$. Quantification of the bright field images of differentiated cells counted per field. $n=3$ biological replicates. The data are represented as Mean \pm SD values from individual subjects. $\left(\mathrm{n}=3\right.$ ) biological replicates, ${ }^{*} P<0.05$ and Kruskal-Wallis one-way ANOVA with Dunn's multiple comparisons (F) Transmission electron microscopy micrograph of ultramicroscopic subcellular structures of the vehicle and IL-1 $\beta(10 \mathrm{ng} / \mathrm{ml}) 24$ h treated hVCF. N: Nucleus; ER: Endoplasmic Reticulum; MV: Secreted Microvesicle; G: Golgi body; M: Mitochondria. Scale bar, 400nm. n=3 technical replicates $(\mathrm{G})$ Schematic representation of cardiac fibroblast 14 days differentiation in T-cell media. hVCF were treated with $\mathrm{rIL}-1 \mathrm{~b}(10 \mathrm{ng} / \mathrm{mL})$ in fibroblast media for 4 days. Cell were then transferred to T-cell expansion media with T-cell activators (CD3, CD2, CD28) for 10 days. Bright field images of cardiac fibroblasts grown in T-cell media with T-cell activators with or without IL-1 $\beta(10 \mathrm{ng} / \mathrm{mL})$ for 13 days. Multicellular clusters marked by arrows were seen in the IL-1 $\beta$ treated wells but not in the vehicle treated wells. Quantification of the multi-cell rosettes clusters is represented as a scatter plot of Mean \pm SD values from individual subjects $(\mathrm{n}=3, * P<0.05$ Kruskal-Wallis one-way ANOVA with Dunn's multiple comparisons). 
Figure 5: Genetic footprints of IL-1 $\beta$ induced $\mathrm{CD4}^{+}$lymphoid cells. Primary hVCF from 3 human donors

(males and female) were differentiated for 10 days in a T-cell media with or without IL-1 $\beta(10 \mathrm{ng} / \mathrm{mL})$ and flow sorted into $\mathrm{CD}^{+}$and $\mathrm{CD} 4-$ populations followed by next generation RNA sequencing using the illumine platform. (A) Heatmaps showing the expression pattern of genes uniquely identified in Veh $\mathrm{CD}^{+}$, Veh CD4IL-1 $\beta \mathrm{CD}^{+}$and IL-1 $\beta$ CD4 $4^{-}$populations. (B) Venn diagram showing the number of common genes among the Veh and IL-1 $\beta$ among the unique gene identified for all the populations. (C) Volcano plot showing the number of significantly upregulated and downregulated genes for each of the comparisons. False Discovery Rate (FDR) (adjusted $P$ value $<0.01$ ). (D) Summary of the gene ontology hits using database for annotation, visualization and integrated discovery (DAVID) online platform showing the enrichment of genes involved in secretion, pluripotency, development, metabolism and T-cell activation and differentiation. Benjamini Hochberg score of false discovery rate $P<0.01$.

Figure 6: IL-1 $\beta$ mediated transdifferentiation of primary human ventricular cardiac fibroblasts (hVCF) into cells with CD4 T-cell markers is through the IL-1R-pMAPK and IL-1R-NFkB pathway. Cultures of primary hVCF from 3 human donors (males and female) were treated with Veh, IL-1 $\beta$ (10 ng/mL), IL-1RA (200 ng/mL) and IL-1 $\beta+$ IL-1RA for 24 h. (A) Quantification of IL-1R gene expression determined using qPCR was performed in primary adult rat cardiac fibroblasts (Left). The data represents Mean \pm SD values from 3 biological replicates. ${ }^{*} P<0.05$ unpaired two tailed student $t$ test. (B) Representative images of IL-1R expression and distribution across the nucleus and cytoplasm of hVCF treated with veh or IL-1 $\beta$. The white dotted lines indicate the bright red foci of IL-1R outside the hVCF after treatment with IL-1 $\beta$. Z stack images of IL-1 $\beta$ treated hVCF were projected in 3D using image J. Arrows indicate overexpression and extracellular release of IL-1R. (C) Bright field images of hVCF differentiation in T-cell media after 14 days of treatment with Veh, IL-1 $(10 \mathrm{ng} / \mathrm{mL})$, IL-1RA (200 ng/mL) or IL-1 +IL-1RA. Quantification of rosette clusters per field performed by a double blinded observer on randomized images. The blacklines indicate the differentiated cell cluster formed in response to IL-1 $\beta$ after 14 days. (D) Phosphorylation of pMAPK Thr180/Tyr182 and 


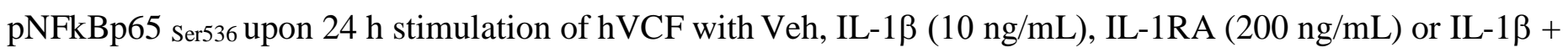

IL-1RA determined using immunoblotting. The data are presented as scatter plot of Mean \pm SD values from individual donors and technical replicates $(\mathrm{n}=3, * P<0.05$ Kruskal-Wallis one-way ANOVA with Dunn's multiple comparisons).

Figure 7: Mechanism underlying cardiac fibrosis based on IL-1ß-induced trans-differentiation. Our results propose a pathway leading to fibrosis in the injured myocardium that encompasses cell plasticity and transdifferentiation. Our data suggest that resident cardiac fibroblasts are activated during hypertensive stress to myofibroblasts and undergo staged polarization/phenotype switching in response to pro-inflammatory signals (such as IL-1 $\beta$ ). This results in the transdifferentiation to a secretory cell with an immune phenotype that participates in the amplification and regulation of the inflammatory response through secretion of immunomodulatory proteins and metabolites. We suggest that the activation of resident fibroblasts may constitute an "emergency response" of the tissue to pressure overload that functions to stabilize and restore tissue before the arrival of macrophages, neutrophils, and other cells from the circulation. This mechanism recognizes the exquisite degree of biological plasticity exhibited by the cardiac fibroblast in mediating repair and regeneration of the myocardium. 


\section{REFERENCES}

1. Price, L.C., et al. Inflammation in pulmonary arterial hypertension. Chest 141, 210-221 (2012).

2. Savai, R., et al. Immune and inflammatory cell involvement in the pathology of idiopathic pulmonary arterial hypertension. Am J Respir Crit Care Med 186, 897-908 (2012).

3. Goldenberg, N.M. \& Steinberg, B.E. Inflammation Drives Pulmonary Arterial Hypertension. Anesthesiology 130, 820-821 (2019).

4. Hassoun, P.M., et al. Inflammation, growth factors, and pulmonary vascular remodeling. J Am Coll Cardiol 54, S10-19 (2009).

5. Dorfmuller, P., Perros, F., Balabanian, K. \& Humbert, M. Inflammation in pulmonary arterial hypertension. Eur Respir J 22, 358-363 (2003).

6. Vergadi, E., et al. Early macrophage recruitment and alternative activation are critical for the later development of hypoxia-induced pulmonary hypertension. Circulation 123, 1986-1995 (2011).

7. Cracowski, J.L., et al. Proinflammatory cytokine levels are linked to death in pulmonary arterial hypertension. Eur Respir J 43, 915-917 (2014).

8. El Kasmi, K.C., et al. Adventitial fibroblasts induce a distinct proinflammatory/profibrotic macrophage phenotype in pulmonary hypertension. J Immunol 193, 597-609 (2014).

9. Zhu, P., Xiong, W., Rodgers, G. \& Qwarnstrom, E.E. Regulation of interleukin 1 signalling through integrin binding and actin reorganization: disparate effects on NF-kappaB and stress kinase pathways. Biochem J 330 ( Pt 2), 975-981 (1998).

10. Caron, J.P., et al. Modulation of matrix metalloprotease 13 (collagenase 3) gene expression in equine chondrocytes by interleukin 1 and corticosteroids. Am J Vet Res 57, 1631-1634 (1996).

11. Tsujino, M., et al. Induction of nitric oxide synthase gene by interleukin-1 beta in cultured rat cardiocytes. Circulation 90, 375-383 (1994).

12. Di Paolo, N.C., et al. Virus binding to a plasma membrane receptor triggers interleukin-1 alpha-mediated proinflammatory macrophage response in vivo. Immunity 31, 110-121 (2009).

13. Lee, W.W., et al. Regulating human Th17 cells via differential expression of IL-1 receptor. Blood 115, 530-540 (2010).

14. Voelkel, N.F., Tuder, R.M., Bridges, J. \& Arend, W.P. Interleukin-1 receptor antagonist treatment reduces pulmonary hypertension generated in rats by monocrotaline. Am J Respir Cell Mol Biol 11, 664-675 (1994).

15. Trankle, C.R., et al. IL-1 Blockade Reduces Inflammation in Pulmonary Arterial Hypertension and Right Ventricular Failure: A Single-Arm, Open-Label, Phase IB/II Pilot Study. Am J Respir Crit Care Med 199, 381-384 (2019).

16. Lonnemann, G., et al. Cytokines in human renal interstitial fibrosis. I. Interleukin-1 is a paracrine growth factor for cultured fibrosis-derived kidney fibroblasts. Kidney Int 47, 837-844 (1995).

17. Xie, T., et al. Single-Cell Deconvolution of Fibroblast Heterogeneity in Mouse Pulmonary Fibrosis. Cell Rep 22, 3625-3640 (2018).

18. Li, M., et al. Emergence of fibroblasts with a proinflammatory epigenetically altered phenotype in severe hypoxic pulmonary hypertension. J Immunol 187, 2711-2722 (2011).

19. Nahrendorf, M. \& Swirski, F.K. Monocyte and macrophage heterogeneity in the heart. Circ Res 112, 1624-1633 (2013).

20. Gomez, D. \& Owens, G.K. Smooth muscle cell phenotypic switching in atherosclerosis. Cardiovasc Res 95, 156164 (2012).

21. Gomez-Arroyo, J., Abbate, A. \& Voelkel, N.F. Pulmonary arterial hypertension and the Enigma code of smouldering inflammation. Eur Respir J 48, 305-307 (2016).

22. Chen, Y., Yang, Z., Zhao, Z.A. \& Shen, Z. Direct reprogramming of fibroblasts into cardiomyocytes. Stem Cell Res Ther 8, 118 (2017).

23. Jayawardena, T.M., et al. MicroRNA-mediated in vitro and in vivo direct reprogramming of cardiac fibroblasts to cardiomyocytes. Circ Res 110, 1465-1473 (2012). 
24. Ramos, G., Hofmann, U. \& Frantz, S. Myocardial fibrosis seen through the lenses of T-cell biology. J Mol Cell Cardiol 92, 41-45 (2016).

25. Yu, Q., Horak, K. \& Larson, D.F. Role of T lymphocytes in hypertension-induced cardiac extracellular matrix remodeling. Hypertension 48, 98-104 (2006).

26. Bansal, S.S., et al. Dysfunctional and Proinflammatory Regulatory T-Lymphocytes Are Essential for Adverse Cardiac Remodeling in Ischemic Cardiomyopathy. Circulation 139, 206-221 (2019).

27. Laroumanie, F., et al. CD4+ T cells promote the transition from hypertrophy to heart failure during chronic pressure overload. Circulation 129, 2111-2124 (2014).

28. Kallikourdis, M., et al. T cell costimulation blockade blunts pressure overload-induced heart failure. Nat Commun 8, 14680 (2017).

29. Suen, C.M., Chaudhary, K.R., Deng, Y., Jiang, B. \& Stewart, D.J. Fischer rats exhibit maladaptive structural and molecular right ventricular remodelling in severe pulmonary hypertension: a genetically prone model for right heart failure. Cardiovasc Res 115, 788-799 (2019).

30. Stenmark, K.R., Meyrick, B., Galie, N., Mooi, W.J. \& McMurtry, I.F. Animal models of pulmonary arterial hypertension: the hope for etiological discovery and pharmacological cure. Am J Physiol Lung Cell Mol Physiol 297, L1013-1032 (2009).

31. Ryan, J., Bloch, K. \& Archer, S.L. Rodent models of pulmonary hypertension: harmonisation with the world health organisation's categorisation of human PH. Int J Clin Pract Suppl, 15-34 (2011).

32. Kim, K. \& Choi, J.H. Involvement of immune responses in pulmonary arterial hypertension; lessons from rodent models. Lab Anim Res 35, 22 (2019).

33. Taraseviciene-Stewart, L., et al. Inhibition of the VEGF receptor 2 combined with chronic hypoxia causes cell death-dependent pulmonary endothelial cell proliferation and severe pulmonary hypertension. FASEB J 15, 427-438 (2001).

34. Nicolls, M.R., et al. New models of pulmonary hypertension based on VEGF receptor blockade-induced endothelial cell apoptosis. Pulm Circ 2, 434-442 (2012).

35. Vang, A., et al. Effect of alpha7 nicotinic acetylcholine receptor activation on cardiac fibroblasts: a mechanism underlying RV fibrosis associated with cigarette smoke exposure. Am J Physiol Lung Cell Mol Physiol 312, L748L759 (2017).

36. Ackers-Johnson, M., et al. A Simplified, Langendorff-Free Method for Concomitant Isolation of Viable Cardiac Myocytes and Nonmyocytes From the Adult Mouse Heart. Circ Res 119, 909-920 (2016).

37. Xia, J., Psychogios, N., Young, N. \& Wishart, D.S. MetaboAnalyst: a web server for metabolomic data analysis and interpretation. Nucleic Acids Res 37, W652-660 (2009).

38. Ali, S.R., et al. Developmental heterogeneity of cardiac fibroblasts does not predict pathological proliferation and activation. Circ Res 115, 625-635 (2014).

39. Kanisicak, O., et al. Genetic lineage tracing defines myofibroblast origin and function in the injured heart. Nat Commun 7, 12260 (2016).

40. Pinto, A.R., et al. Revisiting Cardiac Cellular Composition. Circ Res 118, 400-409 (2016).

41. Moore-Morris, T., Tallquist, M.D. \& Evans, S.M. Sorting out where fibroblasts come from. Circ Res 115, 602-604 (2014).

42. Humbert, M., et al. Increased interleukin-1 and interleukin- 6 serum concentrations in severe primary pulmonary hypertension. Am J Respir Crit Care Med 151, 1628-1631 (1995).

43. Hebel, K., et al. IL-1beta and TGF-beta act antagonistically in induction and differentially in propagation of human proinflammatory precursor CD4+ T cells. J Immunol 187, 5627-5635 (2011).

44. Bujak, M. \& Frangogiannis, N.G. The role of IL-1 in the pathogenesis of heart disease. Arch Immunol Ther Exp (Warsz) 57, 165-176 (2009).

45. Chung, Y., et al. Critical regulation of early Th17 cell differentiation by interleukin-1 signaling. Immunity 30, 576587 (2009).

46. Deknuydt, F., Bioley, G., Valmori, D. \& Ayyoub, M. IL-1beta and IL-2 convert human Treg into T(H)17 cells. Clin Immunol 131, 298-307 (2009). 

available under aCC-BY-NC-ND 4.0 International license.

47. Psarras, S., Beis, D., Nikouli, S., Tsikitis, M. \& Capetanaki, Y. Three in a Box: Understanding Cardiomyocyte, Fibroblast, and Innate Immune Cell Interactions to Orchestrate Cardiac Repair Processes. Front Cardiovasc Med 6, 32 (2019).

48. Van Linthout, S., Miteva, K. \& Tschope, C. Crosstalk between fibroblasts and inflammatory cells. Cardiovasc Res 102, 258-269 (2014).

49. Grebe, A., Hoss, F. \& Latz, E. NLRP3 Inflammasome and the IL-1 Pathway in Atherosclerosis. Circ Res 122, 17221740 (2018).

50. Ranchoux, B., et al. Endothelial-to-mesenchymal transition in pulmonary hypertension. Circulation 131, 10061018 (2015).

51. Siamwala, J.H., et al. Adaptive and innate immune mechanisms in cardiac fibrosis complicating pulmonary arterial hypertension. Physiol Rep 8, e14532 (2020).

52. Sandstedt, J., et al. Human cardiac fibroblasts isolated from patients with severe heart failure are immunecompetent cells mediating an inflammatory response. Cytokine 113, 319-325 (2019).

53. Nakaya, M., et al. Cardiac myofibroblast engulfment of dead cells facilitates recovery after myocardial infarction. J Clin Invest 127, 383-401 (2017).

54. Huertas, A., et al. Leptin and regulatory T-lymphocytes in idiopathic pulmonary arterial hypertension. Eur Respir J 40, 895-904 (2012).

55. Tamosiuniene, R., et al. Regulatory T cells limit vascular endothelial injury and prevent pulmonary hypertension. Circ Res 109, 867-879 (2011).

56. Yano, T., et al. Intracardiac fibroblasts, but not bone marrow derived cells, are the origin of myofibroblasts in myocardial infarct repair. Cardiovasc Pathol 14, 241-246 (2005).

57. Zeisberg, E.M. \& Kalluri, R. Origins of cardiac fibroblasts. Circ Res 107, 1304-1312 (2010).

58. Acharya, A., Baek, S.T., Banfi, S., Eskiocak, B. \& Tallquist, M.D. Efficient inducible Cre-mediated recombination in Tcf21 cell lineages in the heart and kidney. Genesis 49, 870-877 (2011).

59. Kaur, H., et al. Targeted Ablation of Periostin-Expressing Activated Fibroblasts Prevents Adverse Cardiac Remodeling in Mice. Circ Res 118, 1906-1917 (2016).

60. Fu, X., et al. Specialized fibroblast differentiated states underlie scar formation in the infarcted mouse heart. $J$ Clin Invest 128, 2127-2143 (2018).

61. Sayed, N., et al. Transdifferentiation of human fibroblasts to endothelial cells: role of innate immunity. Circulation 131, 300-309 (2015).

62. Weirather, J., et al. Foxp3+CD4+ T cells improve healing after myocardial infarction by modulating monocyte/macrophage differentiation. Circ Res 115, 55-67 (2014).

63. Roy, B., et al. Fibroblast rejuvenation by mechanical reprogramming and redifferentiation. Proc Natl Acad Sci U S A 117, 10131-10141 (2020).

64. Netea, M.G., et al. Defining trained immunity and its role in health and disease. Nat Rev Immuno/ 20, 375-388 (2020).

65. Bonnet, S., et al. The nuclear factor of activated T cells in pulmonary arterial hypertension can be therapeutically targeted. Proc Natl Acad Sci U S A 104, 11418-11423 (2007). 


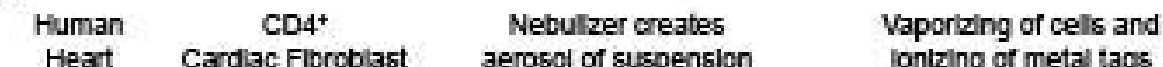
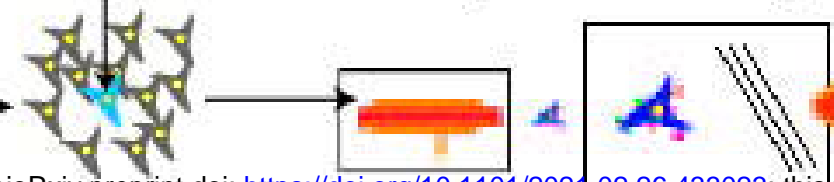

$\because \overline{\bar{F}}$

bioRxiv preprint doi: https://doi.org/10.1101/2021.02.26.433023; this version posted February 26, 2p21. The copyright horder for this preprint (which was not certified by peer review) is the author/funder, who has granted bioRxiv a license to display the preprint in perpetuity. It is made

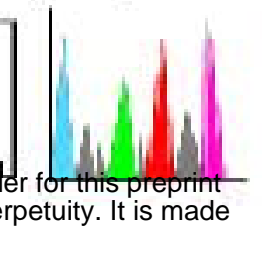

Immune Cell Markers
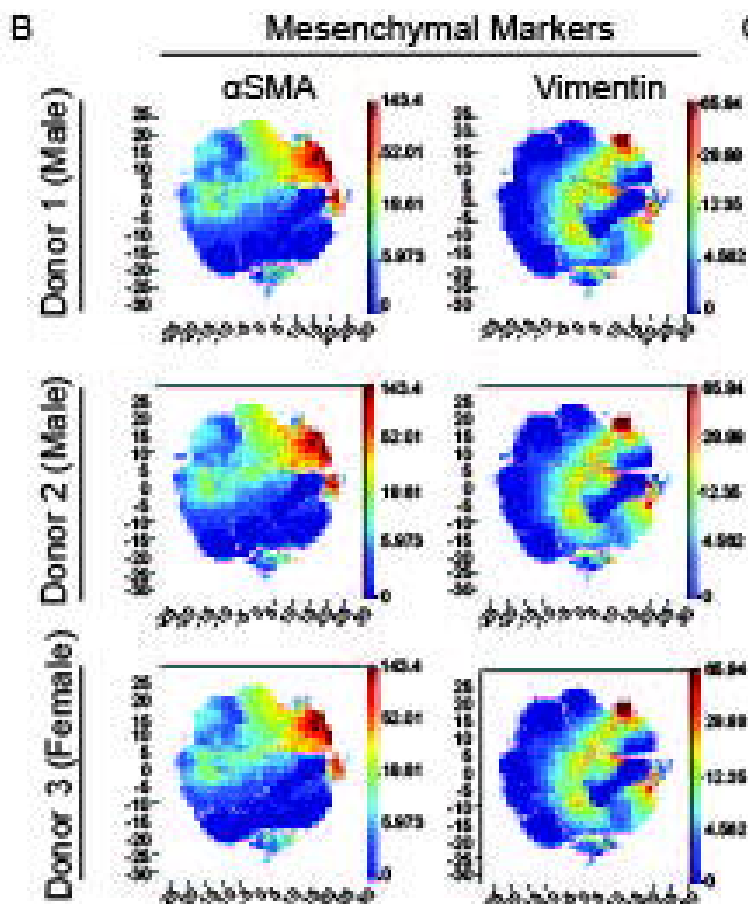

C
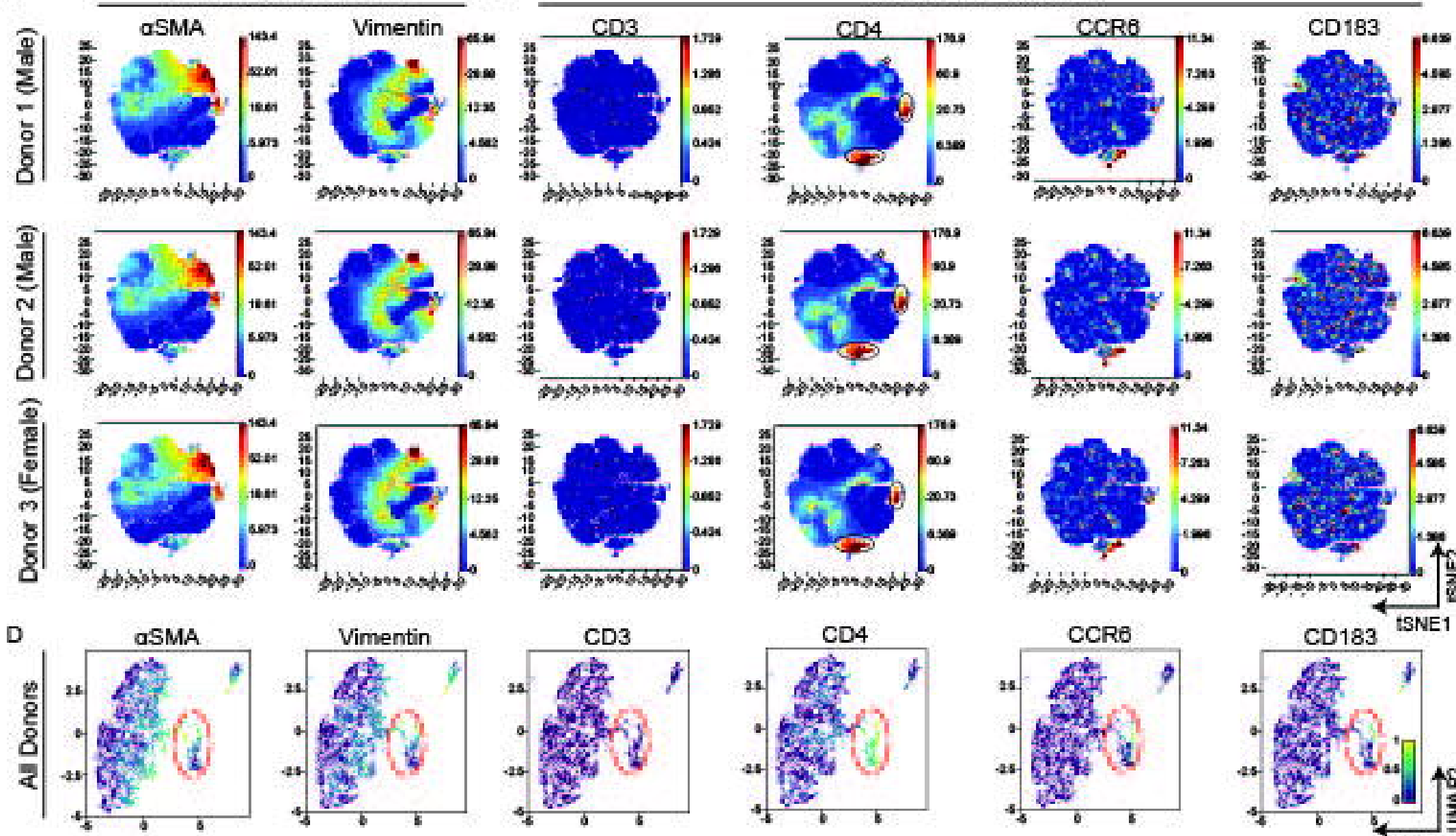

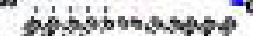
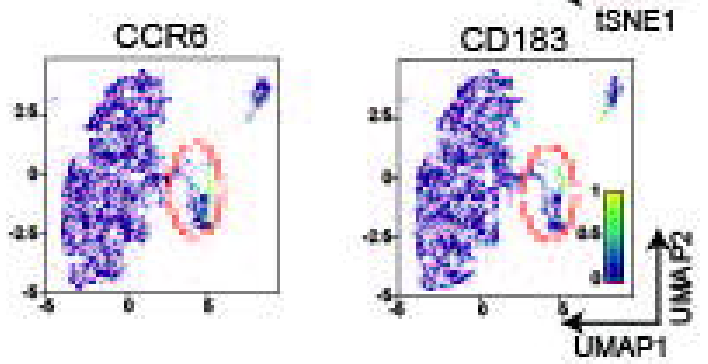

E

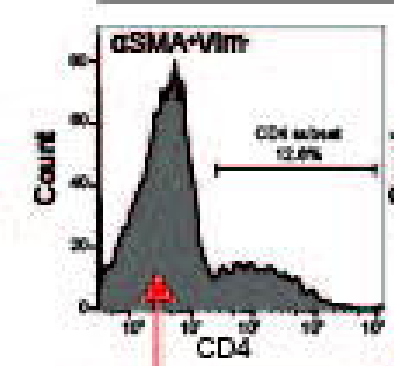

Donor 1 (Male)
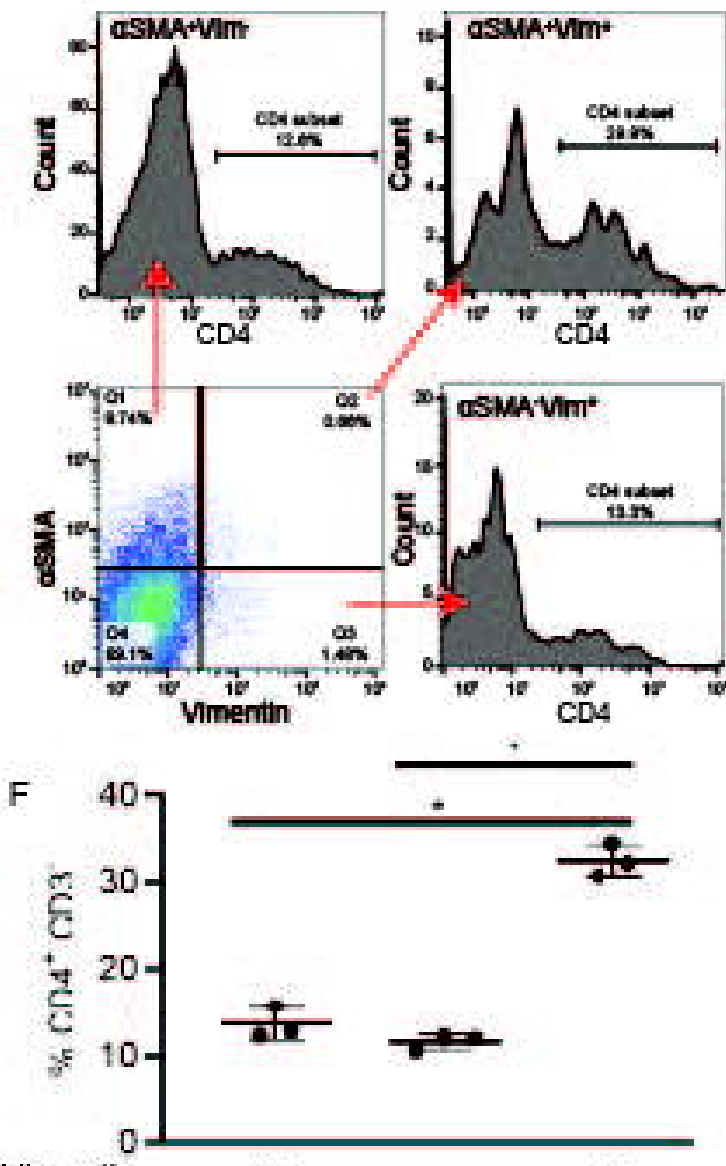

Vimentin
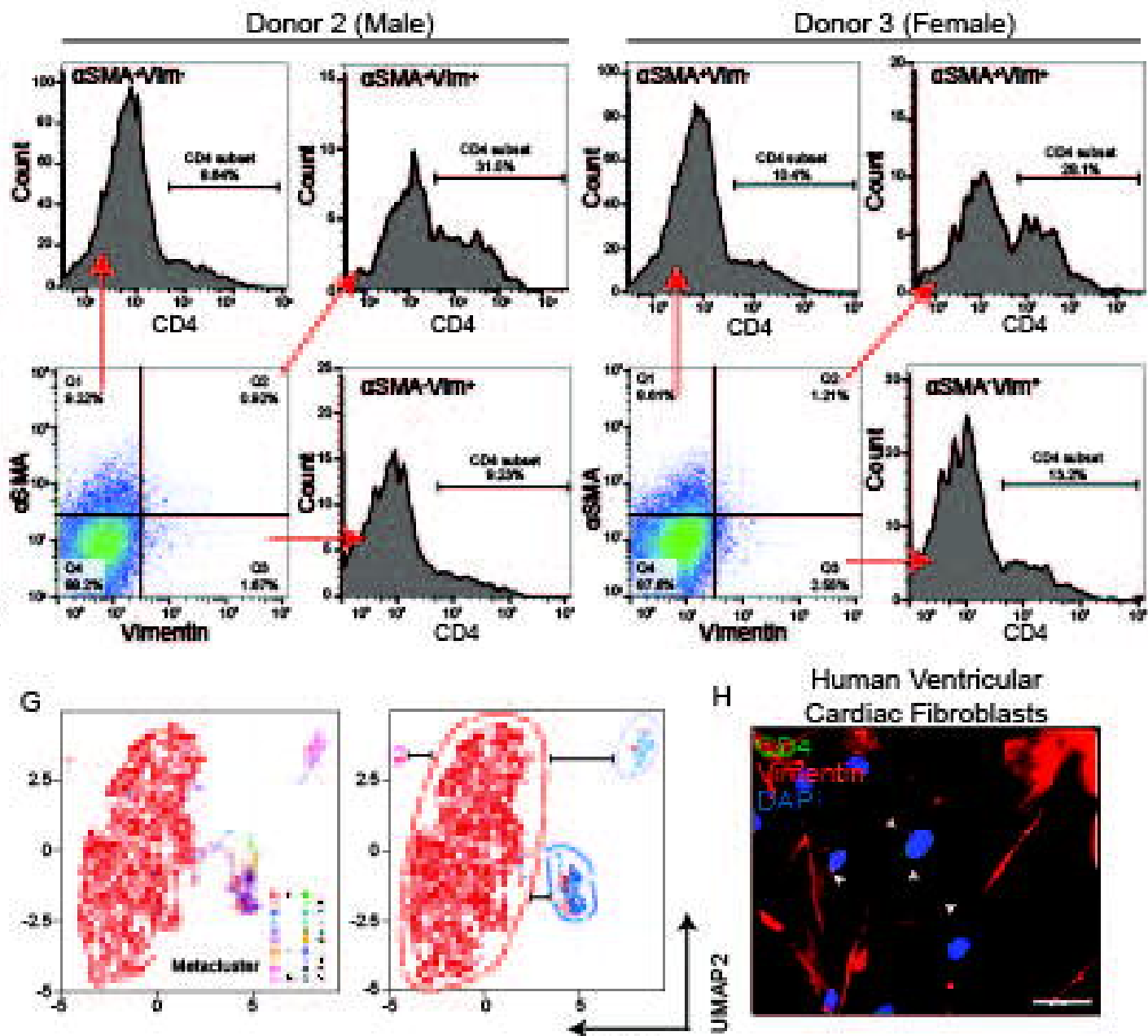

$\mathrm{H}$
Curdian Ventricular

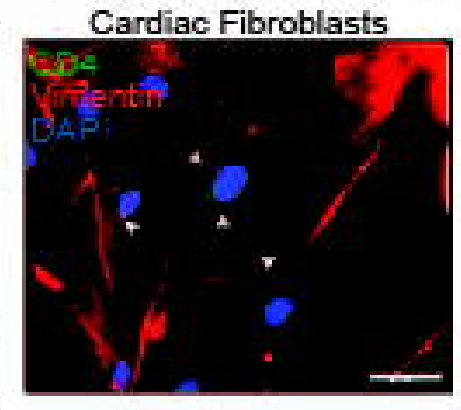


Figure 3

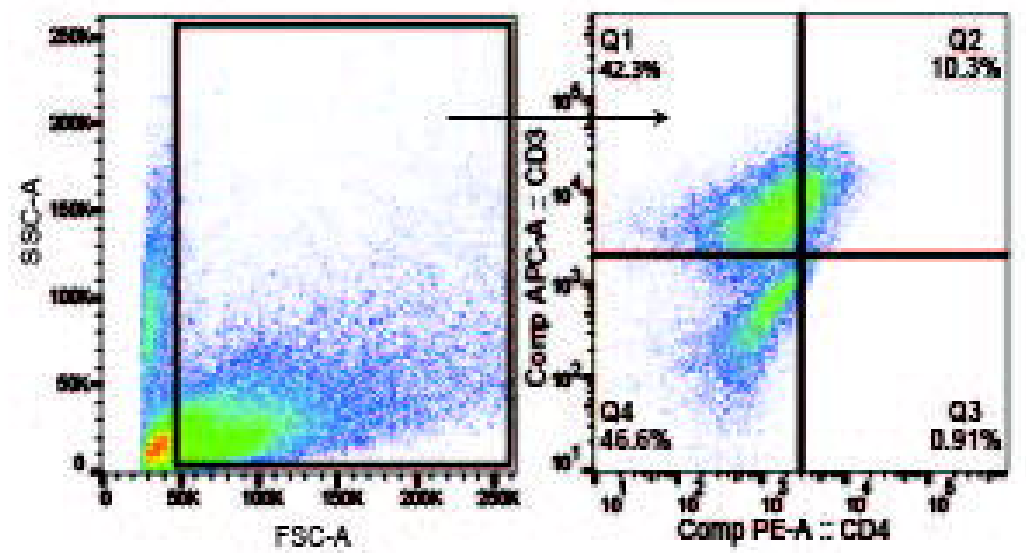

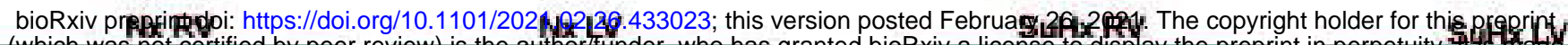
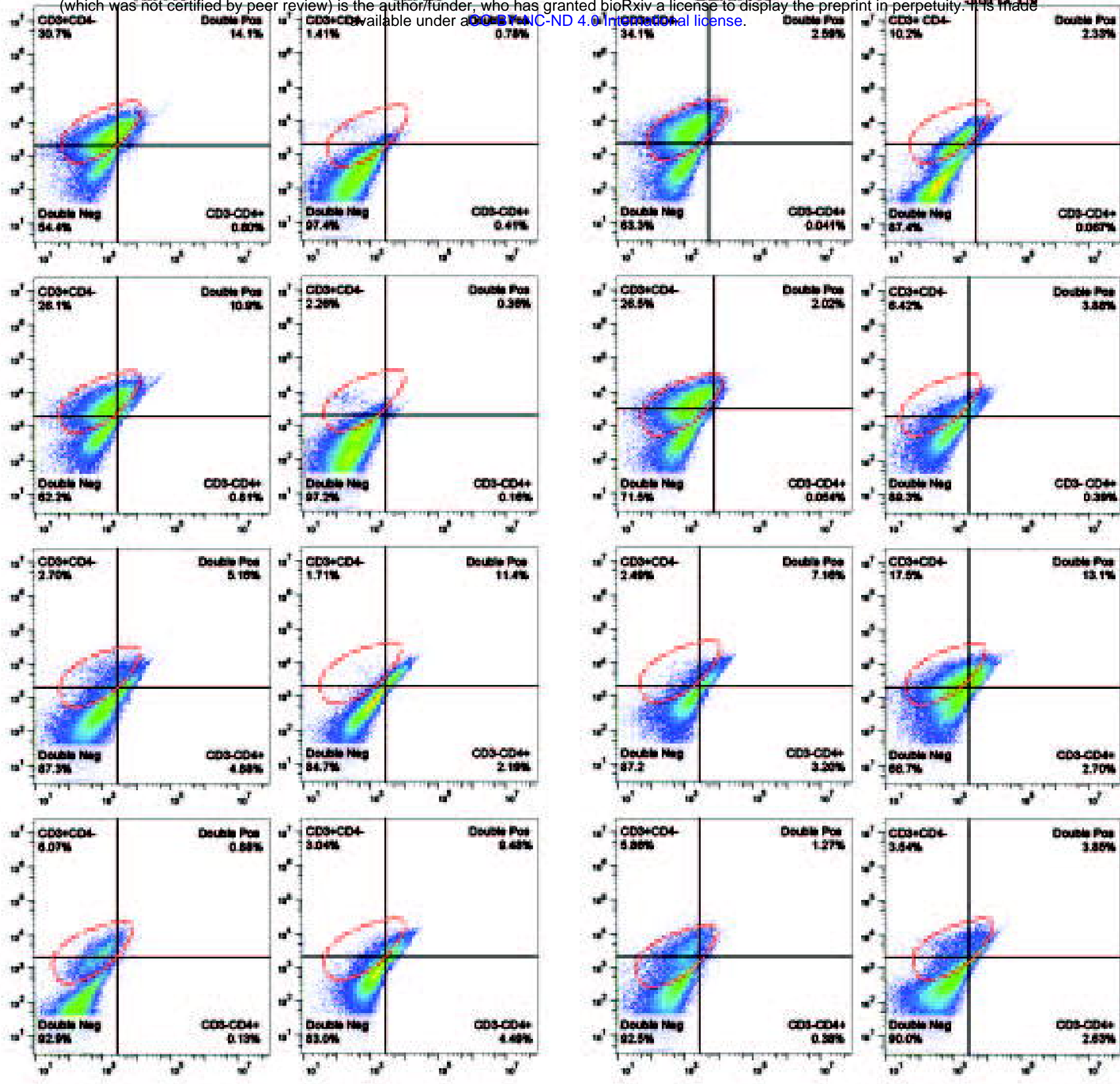

CD4

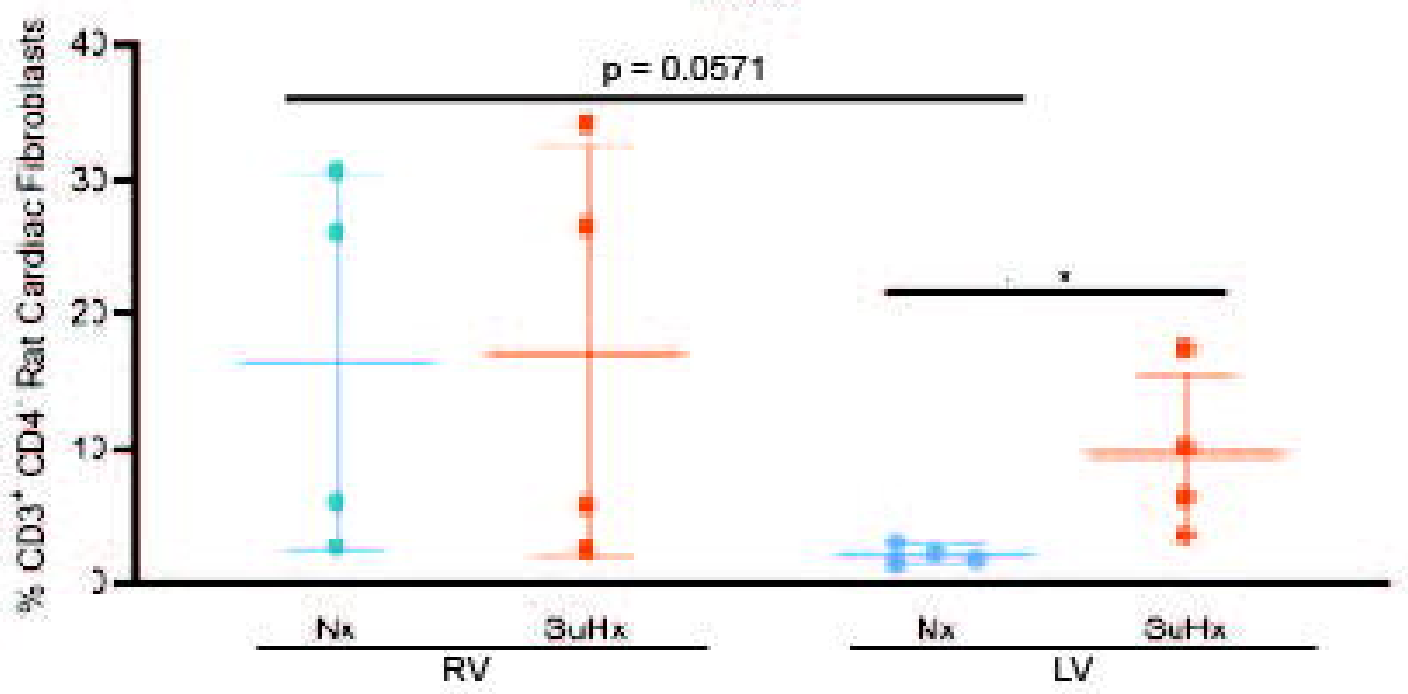


Figure 4

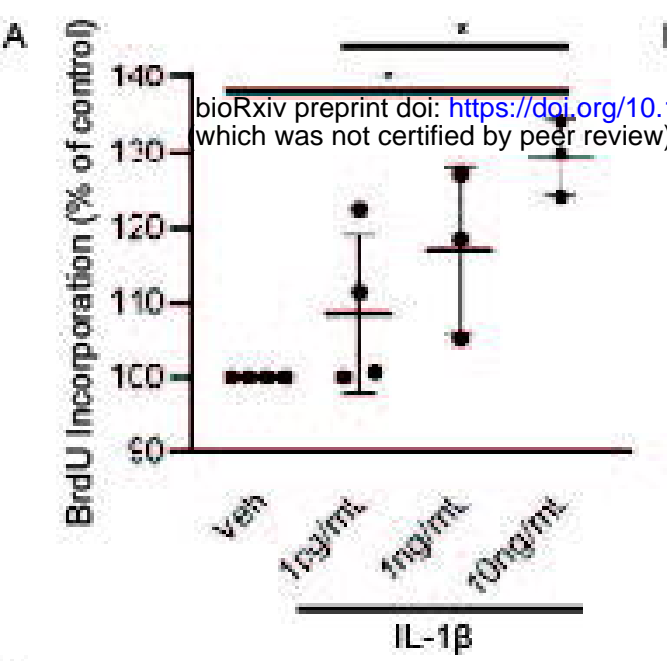

B C
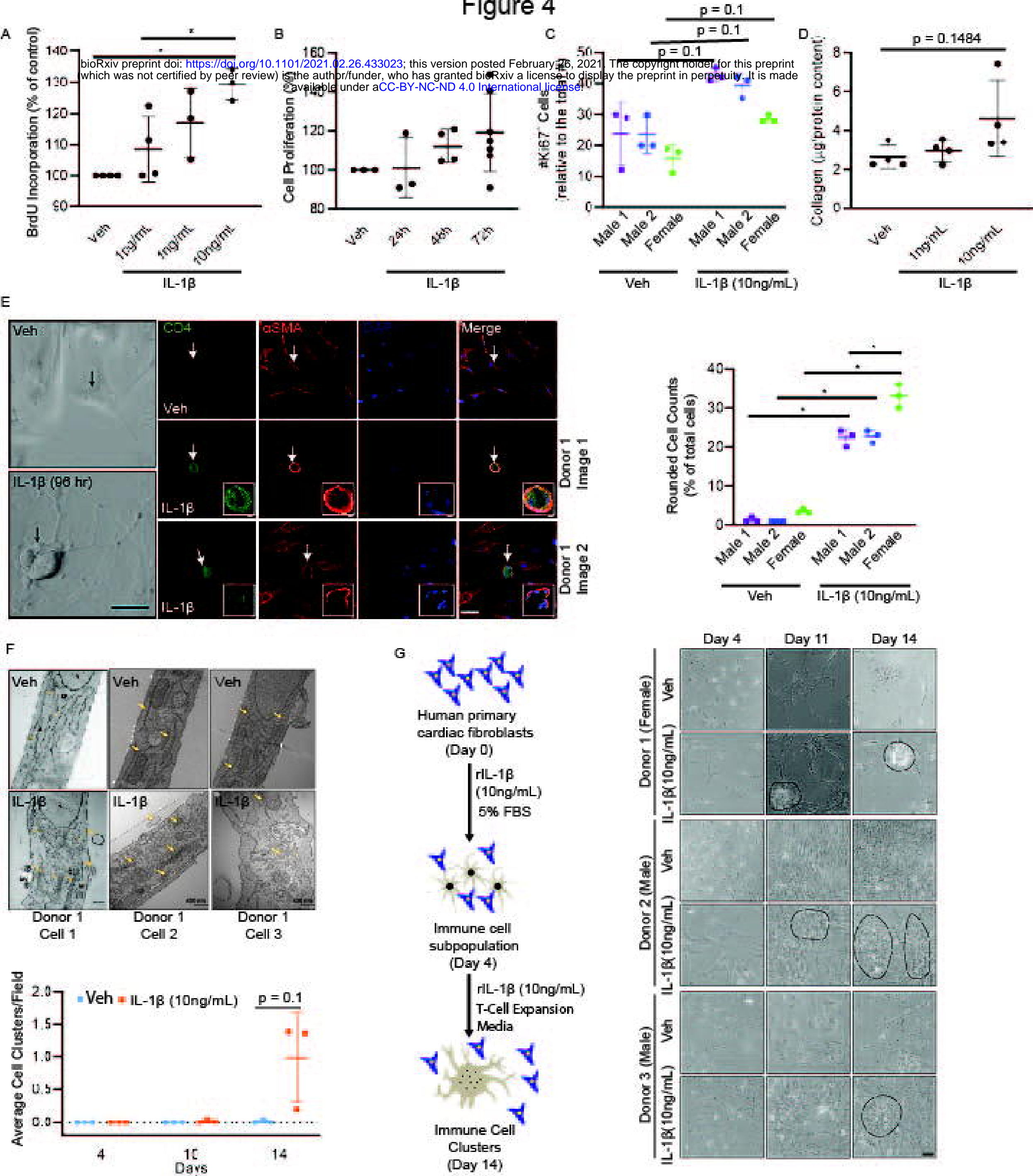

G

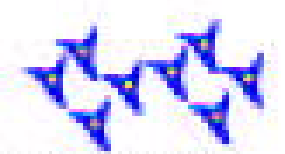

Human primary cardiac fibroblasts

(Day 0)

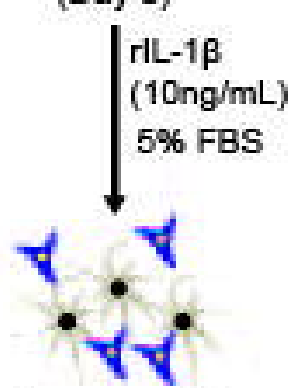

Immune cell subpopulation

(Day 4)

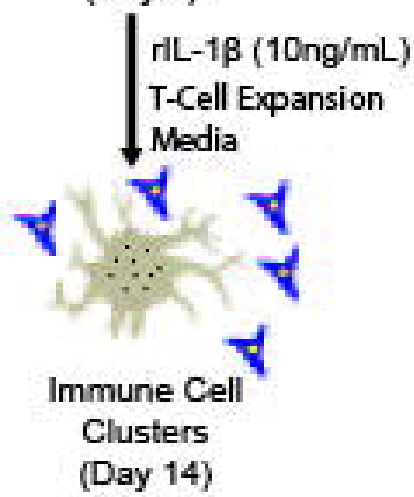

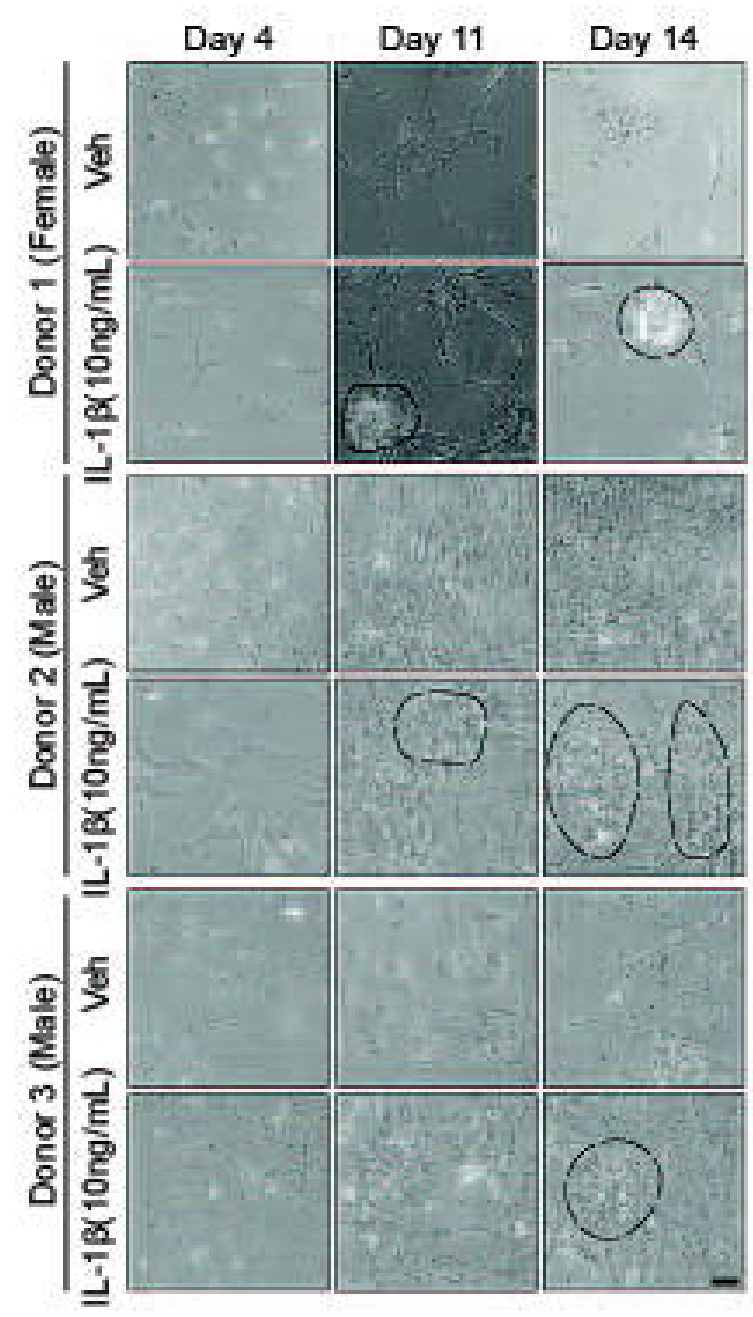


A bioRxiv preprint doi: https://doi.org/10.1101/2021.02.26.433023; this version pested February 26, 2021. The copyright holder for this preprint (which was not certified by peer review) is the author/funder, who has granted bioRxiv a license to display the preprint in perpetuity. It is made

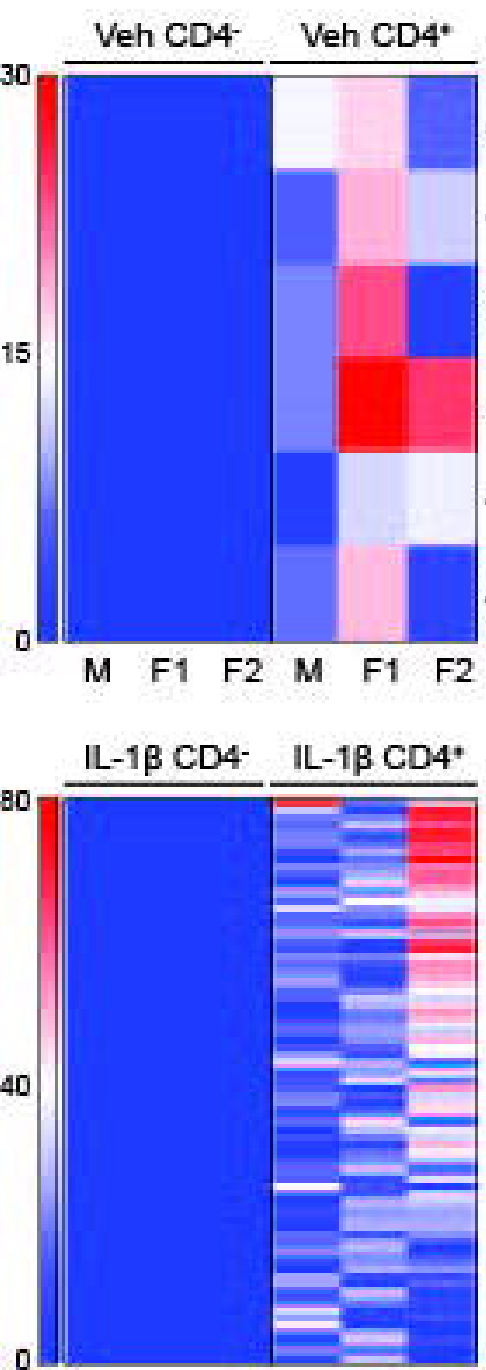

$\begin{array}{llllll}M & F 1 & F & & & \end{array}$ C

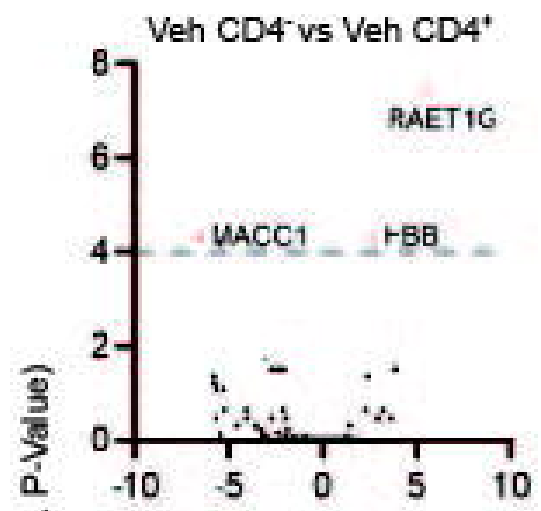

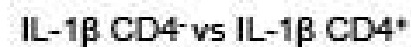

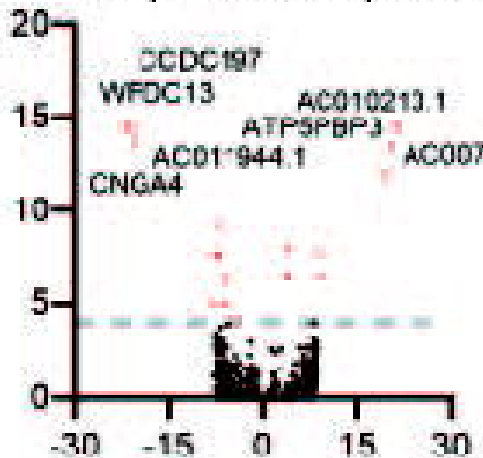

Veh CD4* IL-1B CD4*
GRIN2D

CCR7

REEP2

MACC1

AL355916.1

AL 365203.2

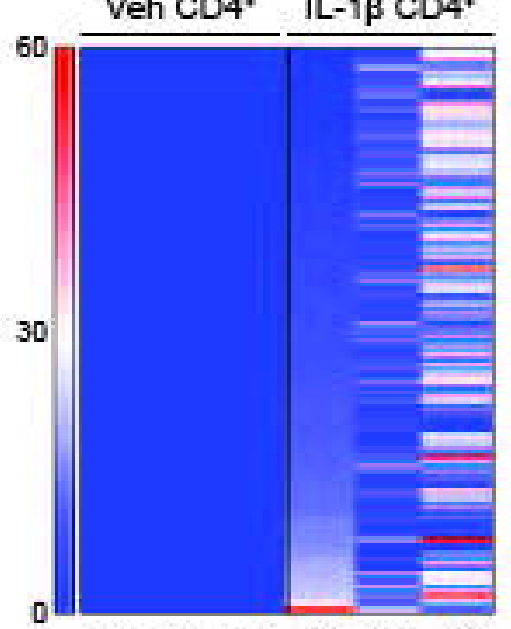

$\begin{array}{llllll}M & F 1 & F 2 & M & F 1 & F 2\end{array}$

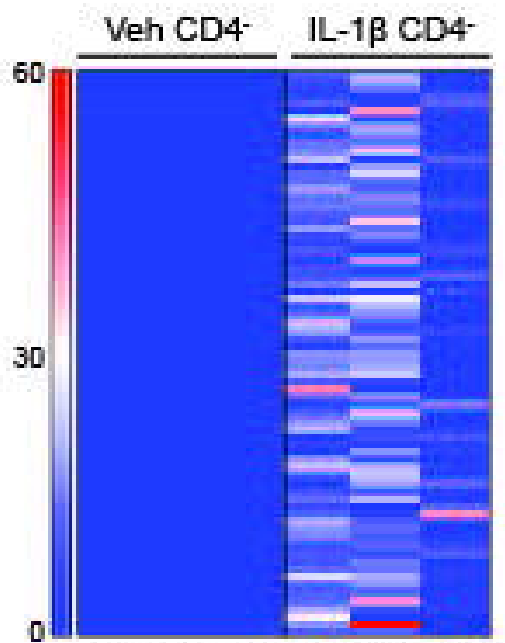

$\begin{array}{llllll}M & F 1 & F 2 & M & F 1 & F 2\end{array}$
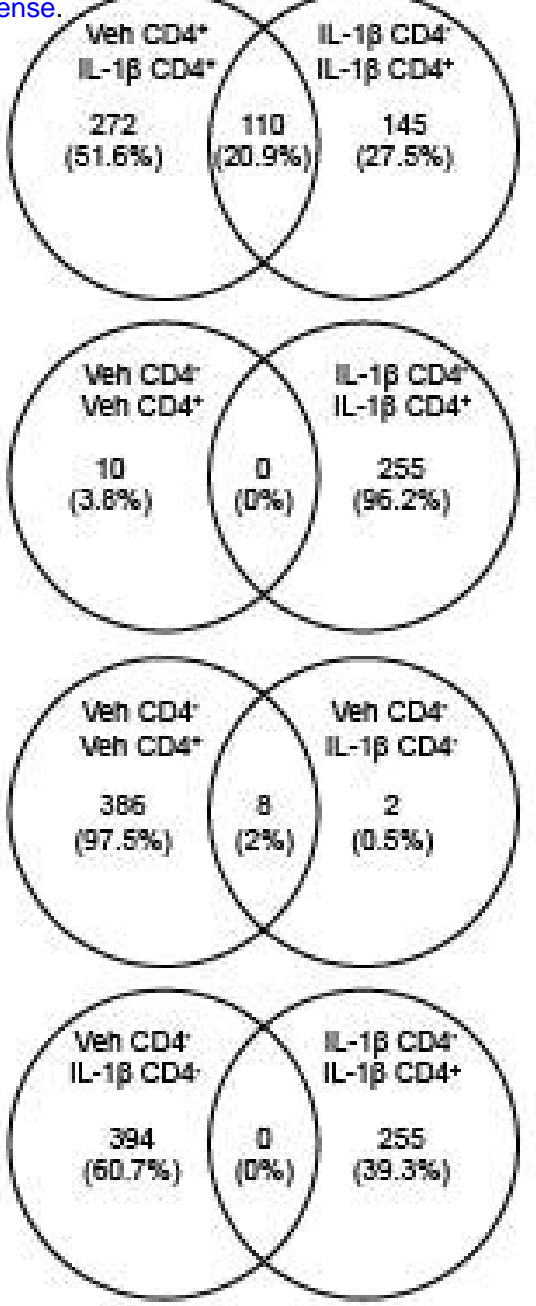

Transdifferentiation

D
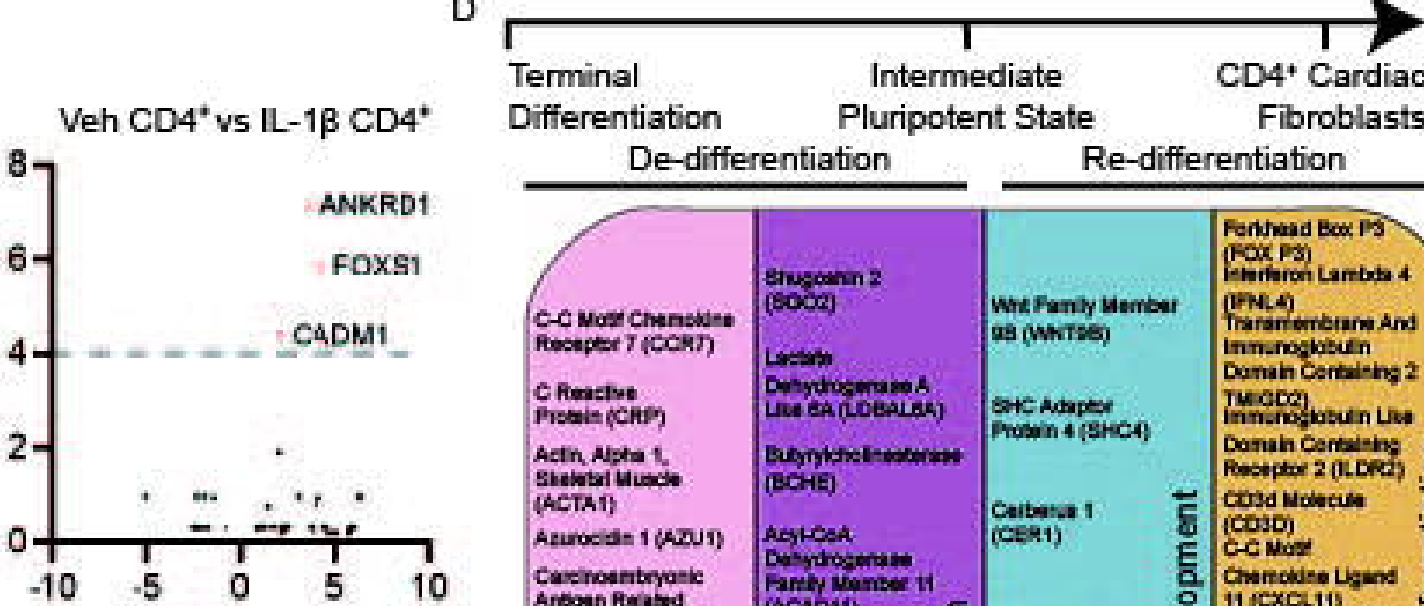

Veh CD4-vs IL-1 13 CD4-

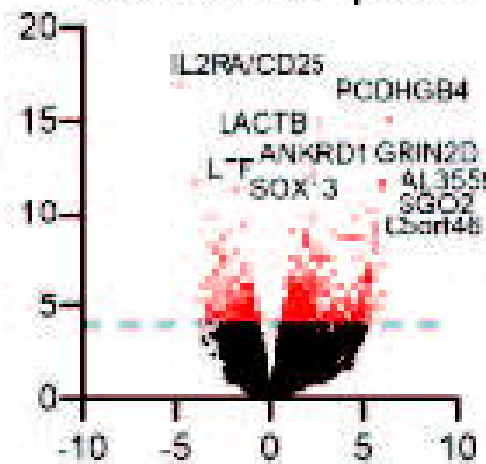

Re-differentiation

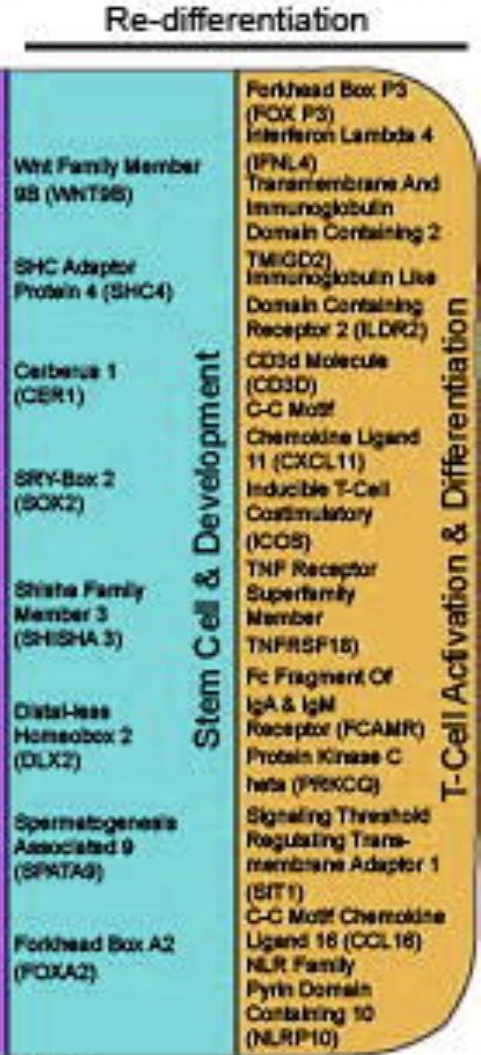

Proliferation 
,

bioRxiv preprint doi: https://doi.org/10.1101/2021.02.26.433023; this
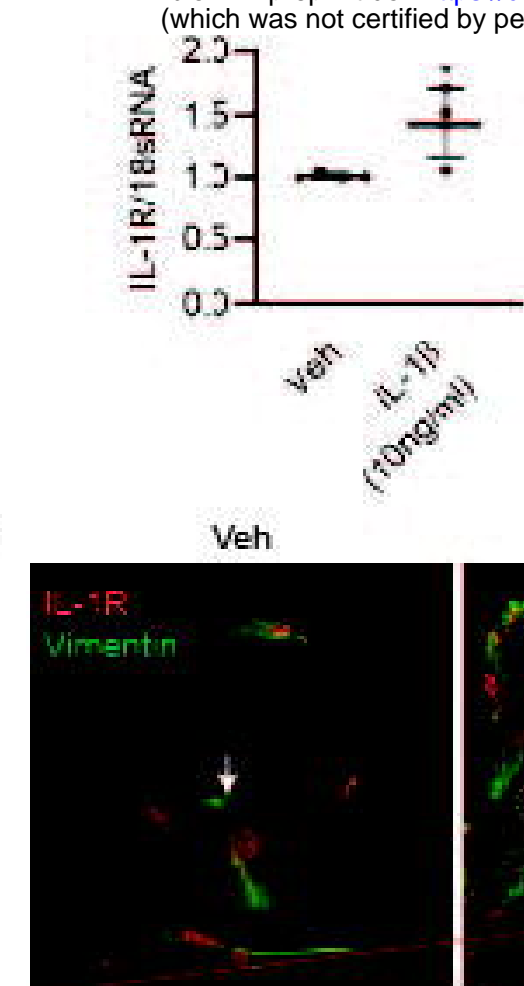

$\mathrm{LL}-1 \beta(10 \mathrm{ng} / \mathrm{mL})$
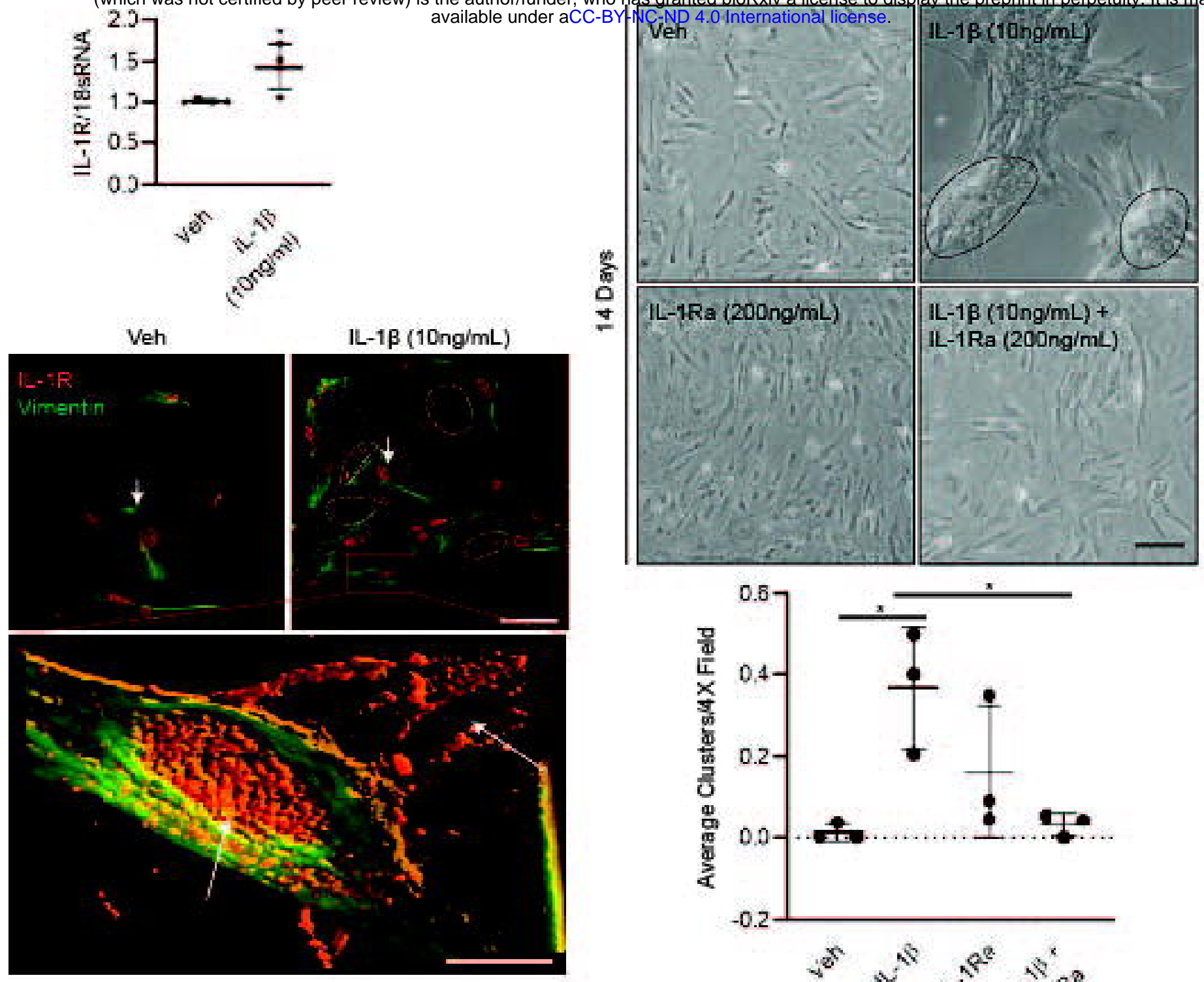

西

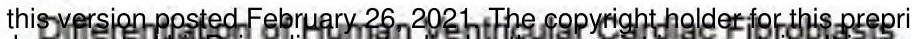

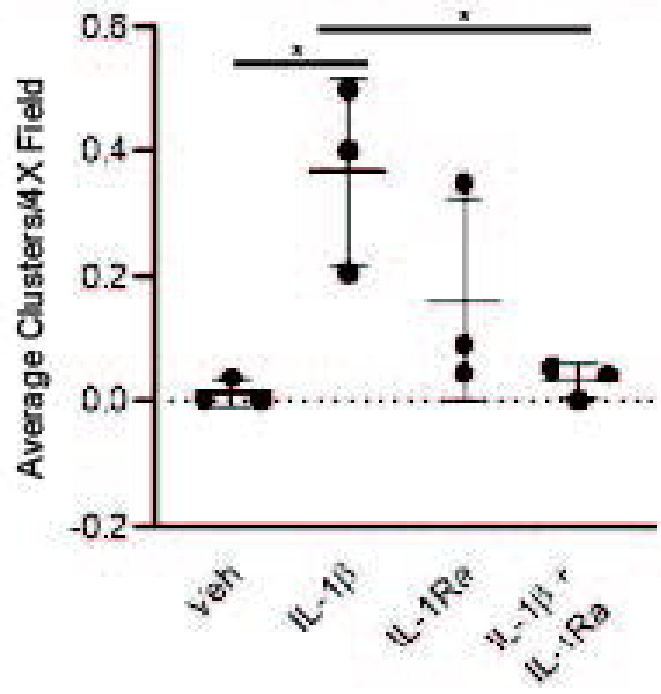

$\mathrm{IL}-1 \beta(10 \mathrm{ng} / \mathrm{mL})-+\quad+$

IL-1Ra (200ng/mL) - - + +

pp38 MAPK $_{\text {messomyras }}=43 \mathrm{kDa}$

tр38 МАPK $\square-\div$ 40kDat

$\mathrm{IL}-1 \beta(10 \mathrm{ng} / \mathrm{mL})-+-+$

IL-1Ra (200ng/mL) - - + +
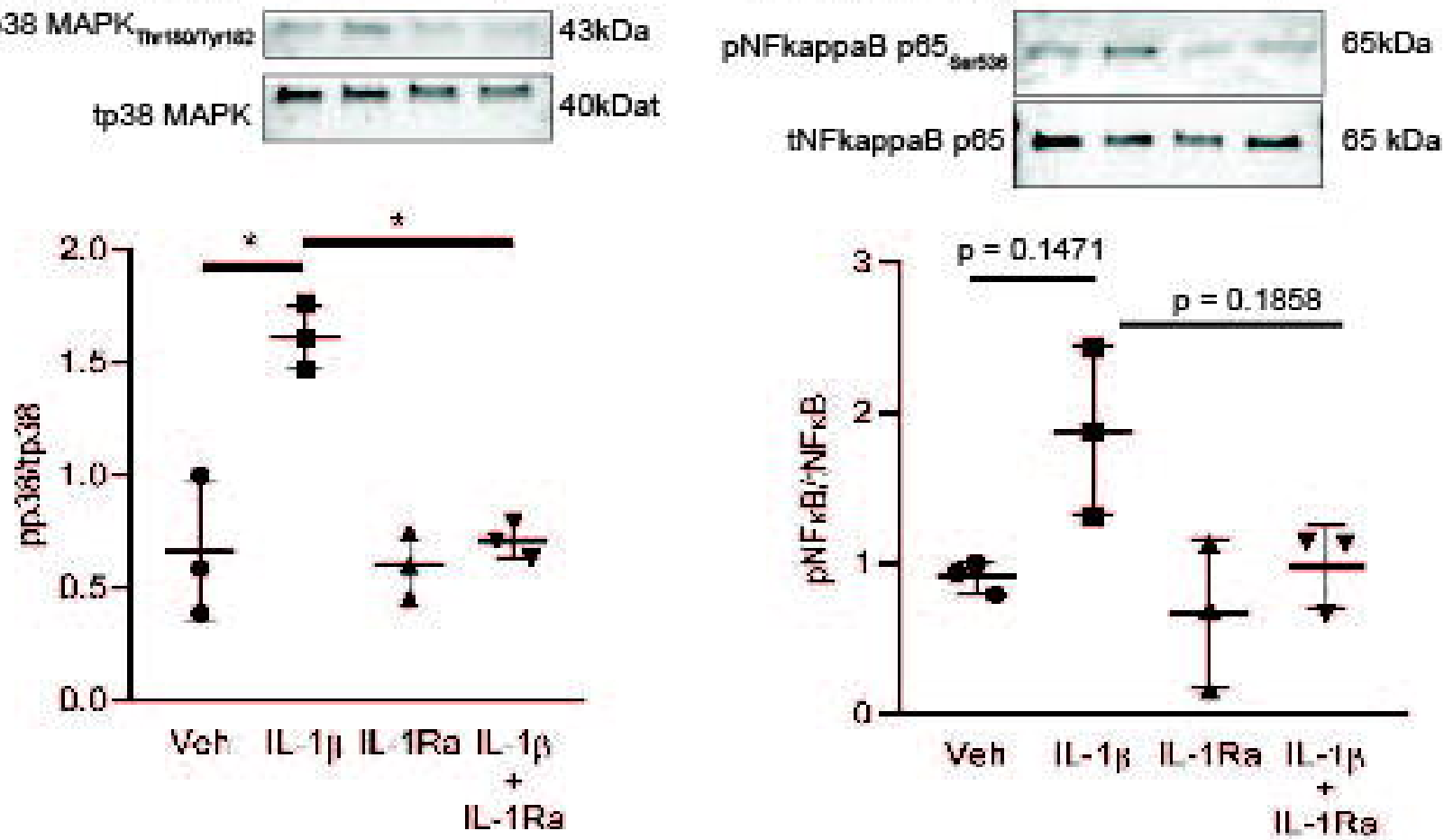
